pi-qg-252

ICMPA-MPA/2011/018

\title{
3D Tensor Field Theory: Renormalization and One-loop $\beta$-functions
}

\author{
Joseph Ben Geloun ${ }^{a, b, \dagger}$ and Dine Ousmane Samary ${ }^{b, \ddagger}$ \\ ${ }^{a}$ Perimeter Institute for Theoretical Physics \\ 31 Caroline St. N., ON, N2L 2Y5, Waterloo, Canada \\ ${ }^{b}$ International Chair in Mathematical Physics and Applications \\ (ICMPA-UNESCO Chair), University of Abomey-Calavi, \\ 072B.P.50, Cotonou, Rep. of Benin
}

E-mails: †jbengeloun@perimeterinstitute.ca, ${ }^{\ddagger}$ ousmanesamarydine@yahoo.fr

\begin{abstract}
We prove that the rank 3 analogue of the tensor model defined in arXiv:1111.4997 [hep-th]] is renormalizable at all orders of perturbation. The proof is given in the momentum space. The one-loop $\gamma$ - and $\beta$-functions of the model are also determined. We find that the model with a unique coupling constant for all interactions and a unique wave function renormalization is asymptotically free in the UV.
\end{abstract}

Pacs numbers: 11.10.Gh, 04.60.-m

Key words: Renormalization, beta-functions, RG flows, tensor models, quantum gravity. 


\section{Introduction}

Approaches to one of the most important problems in physics, namely the quantum gravity (QG) conundrum, have evolved a lot since the last two decades. The most known contender having some undeniable results is certainly String Theory [1] whereas alternative approaches ${ }^{1}$ like Asymptotic Safety scenario [2, Noncommutative Geometry [3, Dynamical Triangulations [4] and Loop Quantum Gravity (LQG) [5] have also drawn a lot of attention of theorists from the mid 90's (see for instance [6] [7] 8] [5]) when, in fact, the String's revolution enchanted most of the physics community.

In the meantime, another framework starts to build up around the Sakharov's idea (1965) of an "emergent" theory of gravity (see [9] for a review). Mainly "emergent" refers to a phenomenon which is only induced and not fundamental. The analogy with hydrodynamics as emerging from laws of molecular physics is commonly used as an illustration. This idea, somehow rooted in condensed matter physics and statistical physics, suggests only, but very originally, that the quantization of the spacetime background metric should be addressed in some independent way than the ordinary study of fluctuations around the flat metric configuration. In fact, it is well known that the latter type of quantization leads inexorably to a non renormalizable quantum field theory using Einstein-Hilbert action [10, 11. So far, results on the QG domain are various and are greeted with more or less success. Nevertheless, the idea of an emergent spacetime was welcome as pertinent and still perpetuates through the years [12.

Coming back to the mid 80's, the so-called random matrix models, initially motivated by string theoretical considerations, become on their own a concrete proposal for statistical models for QG in 2D [13]. Soon after, they were followed by their tensor analogue for $D>2$ QG [14]-[16]. However, the latter works experienced difficulties because one of the main analytical tool, namely the $1 / N$ expansion, was crucially missing. In last resort, only numerical results can be properly achieved. All these endeavors appropriately realize the idea of an emergent theory of gravity (in short, in $2 D$, random matrix models are theories of random triangulations of surfaces and summing on such triangulations amounts to sum over geometries of these surfaces. These models possess a phase transition towards a "continuous phase" geometry the so-called conformal geometry coupled to Liouville gravity [13]).

The framework of tensor fields for addressing the QG issue was correctly stated in [17] for a $D$-dimensional lattice version of 3D Euclidean gravity. The prime idea was not so much focused on the understanding of an extended version of statistical analysis of matrix models for tensors but rather to introduce a discrete version of a simplified and "flat" (BF) model of gravity valid in dimension higher than 2. Substituting the integral over all $D$-dimensional manifolds by a sum over all $D$-dimensional simplicial complexes, the ensuing models generate perturbatively all $D$ simplicial complexes providing the tensor analogues of the successful matrix models. Fields here are defined over several copies of an abstract Lie group which, by Fourier transform, yield tensor components. Depending on the dimension, to each tensor is associated a simplex and vertices provide fusion rules and exchange of momenta as in ordinary quantum field theory. A key point of this formalism is that these tensors should be constrained to satisfy specific rules of invariance enforcing the flatness condition of the

\footnotetext{
${ }^{1}$ We include Supergravity and M-Theory within the String approach as theories invoking extra symmetries or dimensions.
} 
gluing of the simplices at the vertices. A generic link was later found between these lattice models and another fast-growing field: the spin foam models which embody the covariant version of the LQG program [18, 19]. Thus, was born a new line of investigation for QG, the so-called group field theory (GFT) [20, 21]. The partition function of GFT sums over both topologies (dictated by the topology of Feynman graphs as simplicial manifolds) and geometries (encoded in the amplitude of these graphs) of a given manifold. GFT claims to be a theory of quantization "of" spacetime itself [21]. This leads, once again, to the problem of obtaining an emergent spacetime at some proper continuum limit.

The GFT framework appears very appealing for performing quantum field theory computations and, naturally, the question of its renormalizability was first systematically addressed [22, 23]. Many other interrogations arose concerning the GFT formalism. "How to control divergences ?" and "why the most important contributions in the partition function would be of the form of a "large" and "smooth" spacetime like the one, we experience ?" were frequently asked. In fact, all these interrogations could have been only satiated by finding a $1 / N$ expansion for tensor models.

The renormalization program for GFT made its first steps and notable facts have been sorted out concerning power-counting theorems of both ordinary GFTs and more involved GFT models in relationship with 4D QG 24]-28]. In the meantime, a drastic improvement concerning the topology of simplices dual to the Feynman graphs has been proved by Gurau by introducing the colored version of these GFTs [29][30]. In [31], it appears clear that relevant operators of the Laplacian form [32] were missing in the $3 D$ Boulatov GFT action [17] and, so, should be added in all GFT actions before discussing of their renormalizability. The renormalization analysis for these type of GFTs proves to be a computational challenge. Concerning the symmetry analysis of GFTs, some efforts have been made in order to show that they possess a quantum group symmetry [33, that they satisfy peculiar Ward identities [34] and that, associated with translation invariance, there exists a conserved classical energy momentum tensor for the colored GFTs [35].

The real breakthrough of the story occurs with the major discovery by Gurau of the analogue of $1 / N$ expansion both for GFTs and independent identically distributed tensor models (see [36] 38] and more other references therein, [39] for a short review of the subject and [40] for a complete overview of the subject and more developments based on this $1 / N$ expansion). Dominant graphs were identified [41] and are associated with simplices of the sphere topology. Important developments followed: the generalization of the Witt-Virasoro algebra (without central charge) for infinite dimensional tree algebras [42], the tensor generalization of the Ising model in any dimension [43], the determination of the universal character of random tensor models generalizing the Wigner-Dyson law for random matrices [44. Furthermore, based on the above developments and for the first time, a $4 D$ QG model, even though simplified, has been found renormalizable at all order of perturbation theory [45]. Let us readily mention that it is not clear in which sense General Relativity could be recovered from the said model (this is why one calls it simplified). However, it is not hopeless to encounter coordinate invariance and more geometrical properties of the background space emerging through the rich structure of that model [46].

The idea of defining a renormalizable and emergent theory of gravity using the tensor approach has matured through years [46] and belongs to a particular pattern extending progressively the renormalization group $(\mathrm{RG})$ analysis from local graphs like in the $\phi_{4}^{4}$ model, to 
the vector cases for condensed matter systems, then to the matrix cases like in noncommutative field theory [47]-[51] incorporating already nonlocality and, now, to higher dimensional tensor theories. The model of [45] is nonlocal and defined over copies of the compact group $U(1)$ hence does not have infrared (IR) divergences. It is built by first integrating four colors (out of five) in the colored model [29] and choosing the last color to be dynamical with respect to 31 . Considering the $1 / N$ expansion, it is known that only certain contributions in the effective action called "melonic" (this terminology refers to [41]) will be not suppressed in power-counting. It remains to truncate the effective action for obtaining relevant and marginal operators of the melonic kind. After analyzing the divergence degree of an arbitrary graph at a given order of perturbation theory, only graphs determined by particular boundary data but having also a regular internal structure turn out to be divergent. This is the "generalized locality" principle for tensor graphs and only those graphs should be renormalized by standard interpolation moves on external data. Interestingly, a peculiar anomaly arises in the expansion. The authors of [45] interpreted this term as matter defects appearing in that $4 D$ gravity model. Renormalization group ( $\mathrm{RG}$ ) flows have been not computed for the model and these deserve indeed to be understood. As it can be easily realized, the combinatorics of such a model becoming very involved should be cautiously handled. The present contribution is a step towards the computation of the RG flow of that $4 D \mathrm{QG}$.

In this paper, we address the RG flows of coupling constants of a tensor model in $3 D$ related to the above $4 D$ model. This model has been briefly outlined in [45] but its renormalizability has been not yet proved. This is an initial property that we need to investigate before computing any flow. We prove that this $3 D$ tensor model over $U(1)^{3}$ is renormalizable at all orders of perturbation. The proof is thoroughly done in the momentum space. This is in contrast with the renormalizability proof of the anterior $4 D$ model which was performed in the direct space. In the momentum basis, the interaction remain of the same form of as in the direct space yielding factorized graph amplitudes somehow more suitable to perform the different optimizations occurring in the multiscale analysis. Hence, using a different basis also convey to a new perspective on these tensor models in general. In a second part of this paper, we compute the one-loop $\gamma$ - (governing the $\mathrm{RG}$ flow of the wave function renormalization) and $\beta$-functions (governing the $\mathrm{RG}$ flow of interaction coupling constants) and analyze the $\mathrm{RG}$ flows of the different coupling constants. We emphasize that it is not necessary to go beyond one-loop computations since the leading order corrections determine the RG flows of coupling constants if the $\beta$-function is not vanishing at this order. The model obtained by merging all coupling constants to a unique one which is somehow the most natural model proves to be asymptotically free in the UV. This feature might be related to the universal Gaussian behavior of these tensor models discussed in [44].

The plan of this paper is as follows: the next section defines the model and states our two main results. Section 3 is devoted to the multiscale analysis and the achievement of a crude power-counting theorem which will be dissected in Section 4. The renormalization of primitively divergent graphs will be performed in Section 5. The calculations of $\gamma$ - and $\beta$-functions and RG flows of coupling constants are detailed in Section 6. Section 7 gives a summary of our results and an outlook of this work. An appendix gathers further details on some results used through the text. 


\section{Rank 3 tensor model over $\mathrm{U}(1)$}

Let us consider four complex three rank tensor fields over the group $U(1), \varphi^{\mathrm{a}}: U(1)^{3} \longrightarrow \mathbb{C}$. The index $\mathrm{a}=0,1,2,3$ is called color. In Fourier modes, $\varphi^{\mathrm{a}}$ can be expanded as

$$
\varphi^{\mathrm{a}}\left(g_{1}, g_{2}, g_{3}\right)=\sum_{p_{j} \in \mathbb{Z}} \varphi_{\left[p_{j}\right]}^{\mathrm{a}} e^{i p_{1} \theta_{1}} e^{i p_{2} \theta_{2}} e^{i p_{3} \theta_{3}}, \quad \theta_{i} \in[0,2 \pi) \quad \text { and } \quad\left[p_{j}\right]=\left(p_{1}, p_{2}, p_{3}\right)
$$

where the group elements $g_{k}=e^{i \theta_{k}} \in U(1) \cong S^{1}$. From now on, we write $\varphi_{123}^{\mathrm{a}}:=\varphi_{p_{1}, p_{2}, p_{3}}^{\mathrm{a}}$ and define a theory in the momentum space with a first kinetic part regarding the colors $\mathrm{a}=1,2$ and 3 ,

$$
S^{\mathrm{kin} ; 1,2,3}=\sum_{p_{j}} \sum_{\mathrm{a}=1}^{3} \bar{\varphi}_{123}^{\mathrm{a}} \varphi_{123}^{\mathrm{a}}
$$

where the sum in $p_{j}$ is performed over all momenta values, for $j=1,2,3$. We do not assume any symmetry under permutation of arguments of these tensors.

The ordinary colored theory [29] is defined by an interaction which can be read off in momentum space as

$$
S^{\mathrm{int}}=\tilde{\lambda} \sum_{p_{j}} \varphi_{123}^{0} \varphi_{345}^{1} \varphi_{526}^{2} \varphi_{641}^{3}+\overline{\tilde{\lambda}} \sum_{p_{j}} \bar{\varphi}_{123}^{0} \bar{\varphi}_{345}^{1} \bar{\varphi}_{526}^{2} \bar{\varphi}_{641}^{3},
$$

the parameters $\tilde{\lambda}$ and $\overline{\tilde{\lambda}}$ being the coupling constants.

We emphasized here an important point concerning these tensor models. The momentum space is in "exact duality" with the direct space in the following sense: up to an inessential constant (a power of $2 \pi$ coming from spatial integrations), interactions in direct and momentum spaces share exactly the same form. This is contrast with other local theories or even nonlocal theory like in noncommutative field theory [51] for which the vertex possesses a delta function of momentum conservation. Although nothing prevents to perform the renormalization in the direct space, the main point for switching in momentum space is that graph amplitudes get factorized in a different and useful way as we will see.

The polar point here is to take a particular kinetic term with respect to the color 0 such that

$$
S^{\mathrm{kin}, 0}=\sum_{p_{j}} \bar{\varphi}_{123}^{0}\left(\sum_{s=1}^{3} a_{s}\left|p_{s}\right|+m\right) \varphi_{132}^{0},
$$

where $\left|p_{s}\right|$ denotes the absolute value of $p_{s}$ and $a_{s}$ are positive wave-function coupling constants. Hence the field of color 0 is assumed to be propagating. ${ }^{2}$ Note that also, the model described by (4) is slightly different from the direct 3D analogue of [45]. The latter could be only reproduced by fixing all $a_{s}$ to a given value.

We use the same procedure of [42] which mainly performs an integration of the partition function with respect to all colors save one. One gets an effective action for the last tensor

\footnotetext{
${ }^{2}$ Remark that a direct space formulation corresponding to such a momentum space kinetic term can be defined by a reduced operator acting on each strand as $-i \tilde{\partial}_{\theta} \phi(\theta)=\sum_{p \in \mathbb{Z}}|p| \tilde{\phi}(p) e^{i p \theta}$, where $\tilde{\phi}(p)$ is the Fourier mode of $\phi$.
} 


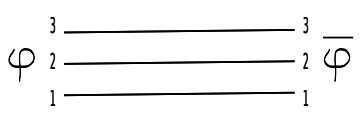

Figure 1: Propagator.

$\varphi^{0}$ in the form

$$
\mathcal{Z}=\int d \mu_{C}\left[\varphi^{0}\right] e^{-S^{\text {int }, 0}}
$$

where $d \mu_{C}\left[\varphi^{0}\right]$ stands for the Gaussian measure with covariance $C=\left(\sum_{s} a_{s}\left|p_{s}\right|+m\right)^{-1}$ (represented in Fig.1) and the effective interaction finds the form

$$
S^{\text {int }, 0}=\sum_{\mathcal{B}} \frac{(\tilde{\lambda} \tilde{\bar{\lambda}})_{\mathcal{B}}}{\operatorname{Sym}(\mathcal{B})} N^{f(p, D)-\frac{2}{(D-2) !} \omega(\mathcal{B})} \operatorname{Tr}_{\mathcal{B}}\left[\bar{\varphi}^{0} \varphi^{0}\right]
$$

the sum in $\mathcal{B}$ is performed on all "bubbles" which are connected vacuum graphs with colors 1 up to $D=3$ and $p$ vertices; $f(p, D)$ is a function of the number of vertices and the dimension; $\omega(\mathcal{B}):=\sum_{J} g_{J}$ is the sum of genera of sub-ribbon graphs called jackets $J$ of the bubble, and $\operatorname{Tr}_{\mathcal{B}}\left[\bar{\varphi}^{0} \varphi^{0}\right]$ are called tensor network operators. Graphs with $\omega(\mathcal{B})=0$ are called melons [40] and non melonic contributions defined by $\omega(\mathcal{B})>0$ become suppressed from (6). Thus, it is sufficient to only focus on the melonic sector of the theory. As in [42], one attributes a different coupling constant to different tensor network operators and simply rewrites (omitting the color index 0)

$$
S^{\text {int }, 0}=\sum_{\mathcal{B}} \frac{\lambda_{\mathcal{B}}}{\operatorname{Sym}(\mathcal{B})} \operatorname{Tr}_{\mathcal{B}}[\bar{\varphi} \varphi]
$$

We truncate the above series and will consider only relevant to marginal terms guided by renormalization conditions. Thus, in the following, we will consider the effective interaction terms made of monomials with order at most four:

$$
S_{4}=\sum_{p_{j}} \varphi_{p_{1}, p_{2}, p_{3}} \bar{\varphi}_{p_{1^{\prime}}, p_{2}, p_{3}} \varphi_{p_{1^{\prime}}, p_{2^{\prime}}, p_{3^{\prime}}} \bar{\varphi}_{p_{1}, p_{2^{\prime}}, p_{3^{\prime}}}+\text { permutations. }
$$

The last term "permutations" means that we include also other terms by performing a permutation over the six momentum arguments (see Fig. 2).

By introducing the UV cutoff $\Lambda$ on the propagator which becomes $C^{\Lambda}$, the action and partition function of our model are then defined by

$$
S^{\Lambda}=\lambda_{4}^{\Lambda} S_{4}+C T_{2 ; 1}^{\Lambda} S_{2 ; 1}+\sum_{s=1}^{3} C T_{s ; 2 ; 2}^{\Lambda} S_{s ; 2 ; 2}, \quad \mathcal{Z}=\int d \mu_{C}^{\Lambda}[\varphi] e^{-S^{\Lambda}}
$$

where

$$
S_{2 ; 1}=\sum_{p_{j}} \bar{\varphi}_{\left[p_{j}\right]} \varphi_{\left[p_{j}\right]}, \quad S_{s ; 2 ; 2}=\sum_{p_{j}} \bar{\varphi}_{\left[p_{j}\right]}\left(\left|p_{s}\right|\right) \varphi_{\left[p_{j}\right]},
$$

where the symbols $C T$ 's are coupling constant counterterms defined by the difference between the renormalized and bare couplings. More precisely, $C T_{2 ; 1}^{\Lambda}$ is a mass counterterm and $C T_{s ; 2 ; 2}^{\Lambda}$ is a wave function counterterm.

The main results of this paper are given by the following statements: 

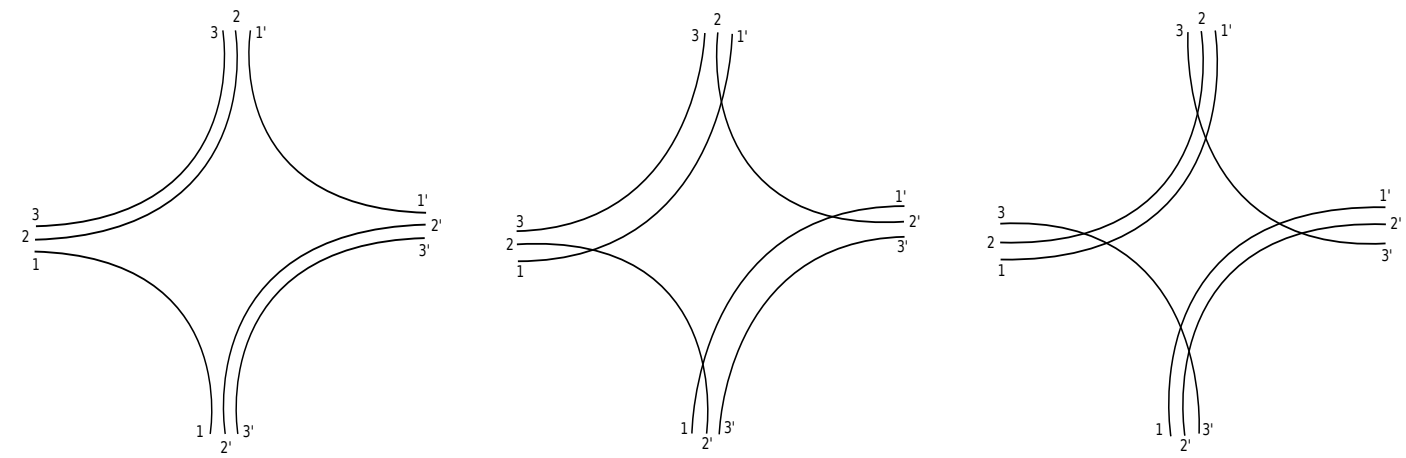

Figure 2: Vertices of the type $V_{4}$.

Theorem 1. The model defined by (9) is renormalizable at all orders of perturbation theory.

Theorem 2. The model obtained from (9) by identifying $a_{s}=a$ is asymptotically free in the UV direction.

\section{Multiscale analysis}

We begin with the multiscale analysis which will lead to a prime (or crude) power-counting theorem. From that theorem, we will be able to identify the "dangerous" graphs for which the renormalization program should be performed. The first step is to find the behavior of the propagator with respect to high and low momentum scales, then, using this result, we should find an optimal way to bound graph amplitudes in the most general manner.

\subsection{Propagator bound}

Consider the kinetic term of the action. The kernel of the propagator is

$$
C\left(\left[p_{s}\right],\left[p_{s}^{\prime}\right]\right)=\left[\sum_{s=1}^{3} a_{s}\left|p_{s}\right|+m\right]^{-1}\left[\prod_{s=1}^{3} \delta_{p_{s}, p_{s}^{\prime}}\right],
$$

the notation $C\left(\left[p_{s}\right],\left[p_{s}^{\prime}\right]\right)$ referring to $C\left(\left[p_{1}, p_{2}, p_{3}\right],\left[p_{1}^{\prime}, p_{2}^{\prime}, p_{3}^{\prime}\right]\right)$.

Introducing a Schwinger parameter, we get the integral form of the propagator as

$$
C\left(\left[p_{s}\right],\left[p_{s}^{\prime}\right]\right)=\int_{0}^{\infty} d \alpha e^{-\alpha\left[\sum_{s=1}^{3} a_{s}\left|p_{s}\right|+m\right]} .
$$

In the following developments, only is needed the small distance or high momenta behavior of that propagator. We do not have the infrared divergence problem from the compactness of $U(1)$. This is reflected in the direct space (Fourier transform of the above), for instance, by the fact that the mass can be put to $m=0$ and this will cause no difficulty with the zero mode of the propagator. Working in the momentum space, in full generality, it is better consider a non vanishing mass otherwise low momenta will cause a divergence in (12). Nevertheless, as mentioned earlier, this situation is actually not relevant for the remaining analysis which solely focuses on the UV sector of the theory. 
The next stage is to introduce the slice decomposition of the propagator in the form:

$$
\begin{aligned}
& C=\sum_{i=0}^{\infty} C_{i} \quad i=1,2 \cdots \\
& C_{0}=\int_{1}^{\infty} d \alpha e^{-\alpha\left[\sum_{s=1}^{3} a_{s}\left|p_{s}\right|+m\right]}, \quad C_{i}=\int_{M^{-i}}^{M^{-i+1}} d \alpha e^{-\alpha\left[\sum_{s=1}^{3} a_{s}\left|p_{s}\right|+m\right]}, \quad M \in \mathbb{N} .
\end{aligned}
$$

The following proposition is direct

Lemma 1. For all $i \in \mathbb{N}$, there exists a large constant $K \geq 0$ such that

$$
C_{i} \leq K M^{-i} e^{-M^{-i}\left|\sum_{s=1}^{3} a_{s}\right| p_{s}|+m|} .
$$

The ultraviolet cutoff can be imposed by summing the slice index $i$ up to a large integer called $\Lambda$ in the slice decomposition (13). Thus

$$
C^{\Lambda}=\sum_{i=0}^{\Lambda} C_{i},
$$

and the ultraviolet limit consists in taking $\Lambda \rightarrow \infty$. One calls $C_{0}$ and $C_{\Lambda}$ the IR and UV propagator slice, respectively. For simplicity in the following, we omit the superscript $\Lambda$.

\subsection{Optimal amplitude bound: Prime power-counting}

Let $\mathcal{G}$ be a connected amputated graph with set of vertices $\mathcal{V}$ (of any kind for the moment) of cardinal $V$ and $\mathcal{L}$ set of lines of cardinal $L=|\mathcal{L}|$. The bare amplitude associated with $\mathcal{G}$ is of the form

$$
\begin{aligned}
A_{\mathcal{G}} & =\sum_{\mu} \frac{f_{\mu}\left[\lambda, C T_{2,1}, C T_{s ; 2 ; 2}\right]}{S(\mathcal{G})} A_{\mathcal{G}, \mu} \\
A_{\mathcal{G}, \mu} & =\sum_{p_{v, s}}\left(\prod_{\ell \in \mathcal{L}} C_{i_{\ell}(\mu)}\left(\left[p_{v(\ell), s}\right] ;\left[p_{v^{\prime}(\ell), s}\right]\right)\right) \prod_{v \in \mathcal{V} ; s} \delta_{p_{v, s}, p_{v^{\prime}, s},}
\end{aligned}
$$

where $f_{\mu}\left[\lambda, C T_{2,1}, C T_{s ; 2 ; 2}\right]$ is a function of some product of coupling parameters, $S(\mathcal{G})$ is a symmetry factor, $p_{v(\ell), s}$ are momenta involved in the propagator which should possess a vertex label $v(\ell)$ hooked to a given line $\ell$ and a strand label $s ; p_{v, s}$ are the same momenta, but now, involved in the vertex which should bear both vertex $v$ and strand $s$ indices; $\delta_{p_{v, s}, p_{v^{\prime}, s}}$ is the Kronecker symbol (we keep here formal notations but, while dealing with a category of vertex $V_{4}, V_{2}$, this symbol might depend on the vertex type and the number of strands $s$ could vary from one category of vertices to the other; however, as we will see below, after some momentum summations, we do not need to track all these indices to get a crude power counting); $\mu=\left(i_{1}, i_{2}, \ldots, i_{q}\right)$ is called momentum assignment and gives to each propagator of each internal line $\ell$ a scale $i_{\ell} \in[0, \Lambda]$; the sum over $\mu$ is performed on all assignments. From the point of view of the effective series expansion [52], the function $f_{\mu}$ collects all the effective couplings corresponding to the attribution $\mu$. Given an amputated graph, we simply have external vertices (where test functions or external fields can be hooked). By 
convention, we fix all external line scales at $i_{\text {ext }}=-1$. $A_{\mathcal{G} ; \mu}$ will be the core quantity and the $\operatorname{sum} A_{\mathcal{G}}=\sum_{\mu}\left[f_{\mu}\left(\lambda, C T_{\ldots}\right) / S(\mathcal{G})\right] A_{\mathcal{G} ; \mu}$ can be done only after renormalization in the standard way of [52].

We would like to perform the momentum sums in the $p_{v, s}$ in an "optimal" way. For that purpose, let us quickly review what should be expected from the ordinary theory. Given $\mu$ and a scale $i$, we consider the complete list of the connected components $G_{i}^{k}, k=1,2, \ldots, k(i)$, of the subgraph $\mathcal{G}_{i}$ made of all lines in $\mathcal{G}$ with the scale attribution $j \geq i$ in $\mu$ (note also that the meaning of $i$ and $k$ are radically different even though we keep simple notations for these quantities when writing $G_{i}^{k}$ ). Such subgraphs are called high or quasi-local and are the key objects in the multiscale expansion [52]. There is a partial (inclusion) order on the set of $G_{i}^{k}$ and $\mathcal{G}_{0}=\mathcal{G}$. The abstract tree made of nodes as the $G_{i}^{k}$ associated to that partial order is called the Gallavotti-Nicolò tree [53]. $\mathcal{G}$ is the root of that tree. To an arbitrary subgraph $g$, one assigns two quantities:

$$
i_{g}(\mu)=\inf _{l \in g} i_{l}(\mu), \quad e_{g}(\mu)=\sup _{l \text { external line of } g} i_{l}(\mu) .
$$

A subgraph $g$ can be viewed as $G_{i}^{k}$ for a given $\mu$ if and only if $i_{g}(\mu) \geq i>e_{g}(\mu)$. In the direct space formalism, one considers a spanning tre $\epsilon^{3} T$ of the graph $\mathcal{G}$. Associated with $T$ are position variables that one integrates to give decay factors to the product of propagator lines. The key point is to optimize the bound over spatial integrations by choosing the tree $T$ to be compatible with the Gallavotti-Nicolò tree. This is achieved by taking the restriction $T_{i}^{k}$ of $T$ to any $G_{i}^{k}$ such that $T_{i}^{k}$ is again a spanning tree for $G_{i}^{k}$.

Coming back to our situation and for simplicity, we assume that no wave function counterterm appears in the graph. Adding them at the end will be an easy task. One notices that the vertex operator is a product of delta functions and hence $A_{\mathcal{G}}$ factorizes in term of closed or open strand line that we call "faces". Let $\mathcal{F}$ be the set of faces of $\mathcal{G}$. It decomposes in a set $\mathcal{F}_{\text {int }}$ of closed (or internal) faces and another set $\mathcal{F}_{\text {ext }}$ of faces connected to external vertices. We have

$$
|\mathcal{F}|=F=F_{\text {int }}+F_{\text {ext }}, \quad F_{\text {int }}=\left|\mathcal{F}_{\text {int }}\right|, \quad F_{\text {ext }}=\left|\mathcal{F}_{\text {ext }}\right|,
$$

and

$$
\begin{aligned}
\left|A_{\mathcal{G} ; \mu}\right| & \leq K^{\prime n} \prod_{\ell \in \mathcal{L}} M^{-i_{\ell}} \sum_{q_{s}} \prod_{\ell \in \mathcal{L}}\left[\prod_{s=1}^{3} \delta_{q_{i_{\ell}}, q_{i_{\ell}}^{\prime}}\right] e^{-M^{-i_{\ell}}\left[\sum_{s} a_{s}\left|q_{s}\right|+m\right]} \\
& \leq K^{\prime n} \prod_{\ell \in \mathcal{L}} M^{-i_{\ell}} \sum_{q_{f}} \prod_{f \in \mathcal{F}} \prod_{\ell \in f} e^{-M^{-i_{\ell}} a_{f}\left|q_{f}\right|}
\end{aligned}
$$

where $a_{f}\left|q_{f}\right|$ is the wave function quantity now bearing a face label $f$. The bound 15 has been also used in order to get (20). The following cases may occur:

(i) The face $f \in \mathcal{F}_{\text {int }}$, then the face amplitude is $\sum_{q_{f}} e^{-\left(\sum_{\ell \in f} M^{-i_{\ell}}\right) a_{f}\left|q_{f}\right|}$. Given $i=$ $\min _{\ell \in f} i_{\ell}$, this amplitude can be optimized as, up to some constant $\delta$,

$$
\sum_{q_{f}} e^{-\left(\sum_{\ell \in f} M^{-i} \ell\right) a_{f}\left|q_{f}\right|} \leq \sum_{q} e^{-M^{-i} a_{f}|q|}=\delta M^{i}+O\left(M^{-i}\right) .
$$

\footnotetext{
${ }^{3}$ This is by definition a subgraph formed by lines passing through all vertices without forming closed loops.
} 
(ii) the face $f$ is open, then all sums in $q_{s}$ can be performed and yield $O(1)$.

Hence, in the above amplitude (20), only terms involving $\mathcal{F}_{\text {int }}$ should be taken into account. We obtain

$$
\begin{aligned}
\left|A_{\mathcal{G} ; \mu}\right| & \leq K^{\prime n} \prod_{\ell \in \mathcal{L}} M^{-i_{\ell}} \sum_{q_{f}} \prod_{f \in \mathcal{F}_{\text {int }}} e^{-\left(\sum_{\ell \in f} M^{-i_{\ell}}\right) a_{f}\left|q_{f}\right|} \\
& \leq K^{\prime n} \prod_{\ell \in \mathcal{L}} \prod_{i=1}^{i_{\ell}} M^{-1} \prod_{f \in \mathcal{F}_{\text {int }}} K^{\prime \prime} M^{i_{l_{f}}}
\end{aligned}
$$

where $l_{f}$ is a strand of $f$ such that $i_{l_{f}}=\inf _{l \in f} i_{l}$. It can be therefore inferred that

$$
\begin{aligned}
\left|A_{\mathcal{G} ; \mu}\right| & \leq K^{\prime n} K^{\prime \prime F_{\mathrm{int}}} \prod_{\ell \in \mathcal{L}} \prod_{i=1}^{i_{\ell}} M^{-1} \prod_{f \in \mathcal{F}_{\mathrm{int}}} \prod_{i=1}^{i_{l_{f}}} M \\
& \leq K^{\prime n} K^{\prime \prime F_{\mathrm{int}}} \prod_{\ell \in \mathcal{L}} \prod_{(i, k) \in \mathbb{N}^{2} / \ell \in G_{i}^{k}} M^{-1} \prod_{f \in \mathcal{F}_{\mathrm{int}}} \prod_{(i, k) \in \mathbb{N}^{2} / l_{f} \in G_{i}^{k}} M \\
& \leq K^{\prime n} K^{\prime \prime F_{\mathrm{int}}} \prod_{(i, k) \in \mathbb{N}^{2}} M^{-L\left(G_{i}^{k}\right)} \prod_{(i, k) \in \mathbb{N}^{2}} \prod_{f \in \mathcal{F}_{\mathrm{int}} \cap G_{i}^{k}} \prod_{l_{f} \in f \cap G_{i}^{k}} M \\
& \leq K^{\prime n} K^{\prime \prime F_{\mathrm{int}}} \prod_{(i, k) \in \mathbb{N}^{2}} M^{-L\left(G_{i}^{k}\right)+F_{\mathrm{int}}\left(G_{i}^{k}\right)},
\end{aligned}
$$

where $L\left(G_{i}^{k}\right)$ and $F_{\text {int }}\left(G_{i}^{k}\right)$ denote the number of internal lines and internal closed faces of $G_{i}^{k}$. In the last step, during the bound optimization, one uses the fact that $l_{f} \in f \cap G_{i}^{k}$ gives a single strand the scale of which is the minimum among the scales of the lines which occur in $f \cap G_{i}^{k}$. Note that the face $f \cap G_{i}^{k}$ can be an open face. In such a case, $l_{f}$ being the minimum of the scales of the closed face $f$ cannot occur in the $f \cap G_{i}^{k}$, hence the product in $l_{f} \in f \cap G_{i}^{k}$ is empty. Thus, the last product yields nothing but the number of elements of the internal closed faces of $G_{i}^{k}$ (when the closed face $f$ becomes totally embedded in the $G_{i}^{k}$ such that $l_{f} \in G_{i}^{k}$; see Fig, 3 ).

Compounding all constant factors coming from the momenta sums, we get a bound of the graph amplitude at a given attribution $\mu$ as

$$
\left|A_{\mathcal{G} ; \mu}\right| \leq K^{n} \prod_{(i, k)} M^{-L\left(G_{i}^{k}\right)+F_{\mathrm{int}}\left(G_{i}^{k}\right)}
$$

where $K$ is some constant and $n$ is the number of vertices of the graph. We recall that the above amplitude is assumed to be without wave-function counterterms. Adding these counterterms in the form of $V_{s ; 2}^{\prime}$ vertices, the following statement is straightforward:

Lemma 2 (Prime power-counting). For a connected graph $\mathcal{G}$ (with external arguments integrated versus fixed smooth test functions), we have

$$
\left|A_{\mathcal{G} ; \mu}\right| \leq K^{n} \prod_{(i, k)} M^{\omega_{d}\left(G_{i}^{k}\right)}
$$



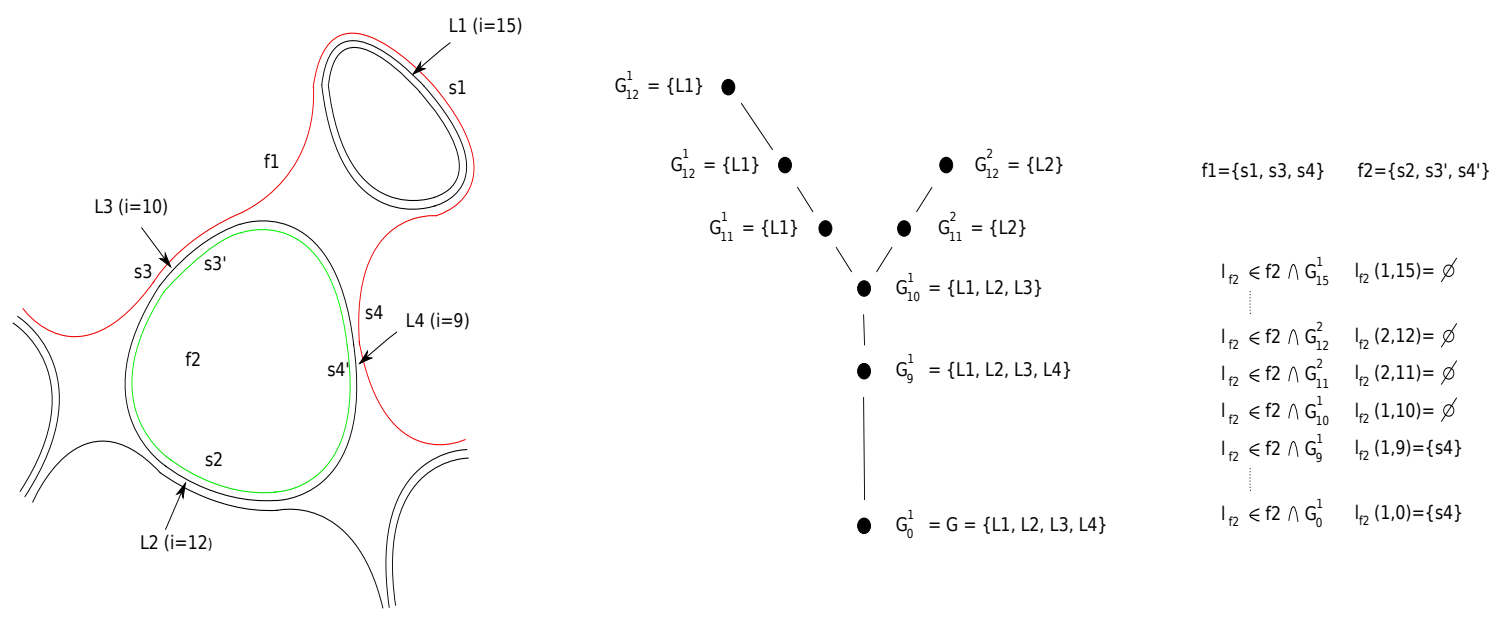

Figure 3: A graph with a multiscale expansion ( $L 1$ is at scale $i=15$, etc.) and its Gallavotti-Nicolò tree; the face $f 1$ (in red) is external and the face $f 2$ (in green) is internal. $l_{f}$ is the strand element at given scale $i$ which should optimize the bound.
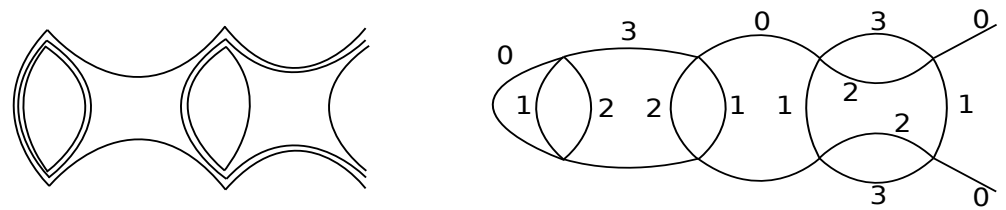

Figure 4: $\quad$ A graph $\mathcal{G}$ (left) and its color extension $\mathcal{G}_{\text {color }}$ (right) in simplified notations: each line of color $\alpha=0,1,2,3$ corresponds to a propagator in the colored theory, i.e. $\int d \mu_{C}(\varphi) \bar{\varphi}_{123}^{\alpha} \varphi_{123}^{\alpha}$, and vertices are defined by Eq.(3).

where $K$ and $n$ are large constants,

$$
\omega_{d}\left(G_{i}^{k}\right)=-L\left(G_{i}^{k}\right)+F_{\text {int }}\left(G_{i}^{k}\right)+\sum_{s=1}^{3} V_{s ; 2}^{\prime}\left(G_{i}^{k}\right) .
$$

The quantity $\omega_{d}(\mathcal{G})$ is called the divergence of the graph $\mathcal{G}$ and provides also the powercounting. In the sequel, we aim at analyzing this quantity for a general connected graph $\mathcal{G}$.

\section{Analysis of the divergence degree}

The analysis of the divergence degree is made in two steps: the first by translating $\omega_{d}(\mathcal{G})$ in topological quantities and the second by refining the obtained result in a convenient manner. From these two steps, we provide an exhaustive list of divergent graphs.

\subsection{Power-counting in topological terms}

We recall some definitions (see [41] [54] 45]): 

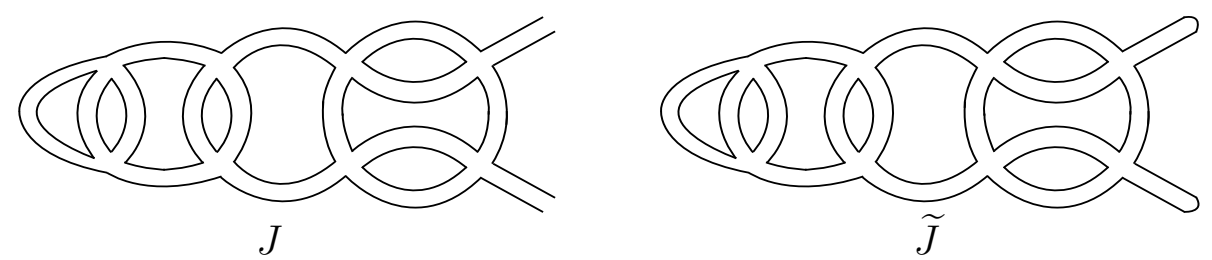

Figure 5: The jacket $J$ (0123), ribbon subgraph of the colored graph $\mathcal{G}_{\text {color }}$ (Fig, 4 and the pinched jacket $\widetilde{J}$ associated with $J$.

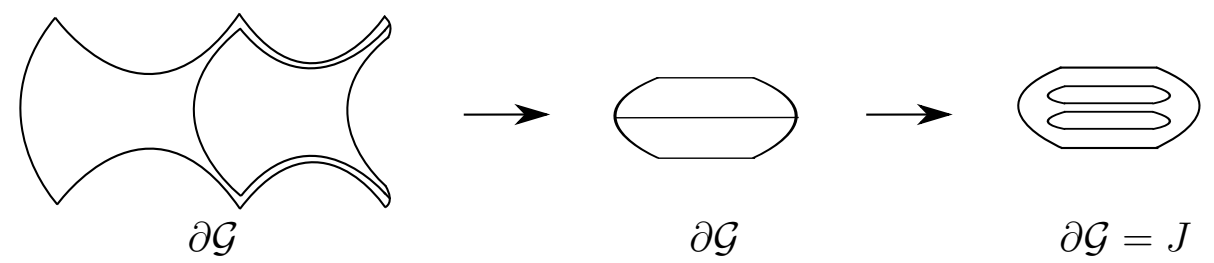

Figure 6: The boundary $\partial \mathcal{G}$ of $\mathcal{G}$ and its rank two or ribbon structure.

Definition 1. Consider $\mathcal{G}$ a 3 dimensional graph.

(i) The colored extension of $\mathcal{G}$ is the unique graph $\mathcal{G}_{\text {color }}$ obtained after restoring in $\mathcal{G}$ the former colored theory graph (Definition 1 in [45], here illustrated in dimension 3 in Fig.4).

(ii) A jacket $J$ of $\mathcal{G}_{\text {color }}$ is a ribbon subgraph of $\mathcal{G}_{\text {color }}$ defined by a cycle $(0 a b c)$ up to a cyclic permutation (see Definition 1 of [41] and an illustration given by Fig.5). There are 3 such jackets due to the dimension 3 .

(iii) The jacket $\widetilde{J}$ is the jacket graph obtained from J after "pinching" that is the procedure consisting in closing all external legs present in J (see Section 3.2 of [54], for the general definition of "pinching" for external strands; here the pinching of a jacket is illustrated in Fig.5). It is always a vacuum graph.

(iv) The boundary $\partial \mathcal{G}$ of the graph $\mathcal{G}$ is the closed graph defined by vertices corresponding to external legs and by lines corresponding to external strands of $\mathcal{G}$ (see Section 3.2 of [54] as well as a drawing in Fig.6]). Here, it is a vacuum graph of the 2 dimensional colored theory, hence a ribbon graph. It is also its own and unique jacket.

Some comments are in order. In general, the boundary graph of a tensor graph of rank $D$ is a closed tensor graph or rank $D-1$. The notion of jacket for the boundary graph can be defined as in the ordinary situation as a cycle of colors. The fact that the boundary graph is defined as a closed graph induces immediately that all jackets are closed.

Let $\mathcal{G}$ be a graph. Let $V_{4}$ be its number of $\varphi^{4}$ vertices, $V_{2}$ the number of vertices corresponding to mass-counterterms $\varphi^{2}, V_{s ; 2}^{\prime}$ the number of vertices corresponding to wave function counterterms $\left|p_{s}\right| \varphi^{2}$. The graph possesses also $L$ number of lines and $N_{\text {ext }}$ external fields. Using the above notations, the following statement holds:

Theorem 3. The divergence degree of connected graph $\mathcal{G}$ is given by

$$
\omega_{d}(\mathcal{G})=-V_{2}-\frac{1}{2}\left(N_{e x t}-4\right)-\sum_{J} g_{\widetilde{J}}+g_{\partial \mathcal{G}}-\left(C_{\partial \mathcal{G}}-1\right)
$$


where the sum is performed on all jackets $J$ of $\mathcal{G}_{\text {color }}, g_{\tilde{J}}$ is the genus of the jacket $\tilde{J}$ associated with $J, g_{\partial \mathcal{G}}$ is the genus of $\partial \mathcal{G}$ and $C_{\partial \mathcal{G}}$ is the number of connected components of the boundary graph $\partial \mathcal{G}$.

Proof. There is a relation between the numbers of lines, of external legs and of vertices for $\mathcal{G}: 4 V_{4}+2\left(V_{2}+\sum_{s=1}^{3} V_{s ; 2}^{\prime}\right)=2 L+N_{\text {ext }}$. Concerning $\mathcal{G}_{\text {color }}$, its number of vertices $V_{\mathcal{G}_{\text {color }}}$ and number of lines $L_{\mathcal{G}_{\text {color }}}$ satisfy

$$
V_{\mathcal{G}_{\text {color }}}=4 V_{4}+2\left(V_{2}+\sum_{s=1}^{3} V_{s ; 2}^{\prime}\right), \quad L_{\mathcal{G}_{\text {color }}}=L+L_{\text {int } ; \mathcal{G}_{\text {color }}}=\frac{1}{2}\left(4 V_{\mathcal{G}_{\text {color }}}-N_{\text {ext }}\right),
$$

where $L_{\text {int } ; \mathcal{G}_{\text {color }}}$ denotes the number of internal lines of $\mathcal{G}_{\text {color }}$ which do not appear in $\mathcal{G}$. $F_{\mathcal{G}_{\text {color }}}$, the number of faces of $\mathcal{G}_{\text {color }}$, can be partitioned in the number $F$ of faces of the initial graph but also in additional faces $F_{\text {int; } ; \text { color }}$ proper to the colored graph and not appearing in $\mathcal{G}$. We write

$$
F_{\mathcal{G}_{\text {color }}}=F+F_{\text {int }} ; \mathcal{G}_{\text {color }} .
$$

There are 3 jackets in $\mathcal{G}_{\text {color }}$. Each face of the graph $\mathcal{G}_{\text {color }}$ is shared by 2 jackets. Summing over the jackets, one therefore has $\sum_{J} F_{J}=2 F_{\mathcal{G}_{\text {color }}}$. Meanwhile, concerning number of vertices and lines, we have $V_{J}=V_{\mathcal{G}_{\text {color }}}$ and lines $L_{J}=L_{\mathcal{G}_{\text {color }}}$, respectively.

The next stage is to pass to topological numbers associated with the graphs. The Euler characteristic of a ribbon graph can be only defined if the graph is closed. This is the reason why we need to consider pinched jackets $\widetilde{J}$. After closing all external half-lines of open jacket graphs $J$, we are in presence of closed ribbon graphs $\widetilde{J}$ for which the above topological number is well defined. For the resulting jacket $\widetilde{J}$, we have

$$
V_{J}=V_{\widetilde{J}}, \quad L_{J}=L_{\widetilde{J}},
$$

hence $\widetilde{J}$ has the same number of vertices, the same number of lines as $J$, but a different number of faces. The number $F_{\widetilde{J}}$ of faces of $\widetilde{J}$ finds the decomposition $F_{\widetilde{J}}=F_{\text {int; } \widetilde{J}}+F_{\text {ext } ; \widetilde{J}}$, where $F_{\text {int; } \widetilde{J}}$ is equal to $F_{\text {int; } J}$ the number of internal faces of $J$ and $F_{\text {ext } ; \widetilde{J}}$ is the number of additional closed faces entailed by the pinching procedure.

The Euler characteristic of $\widetilde{J}$ affords

$$
F_{\text {int } ; \widetilde{J}}+F_{\text {ext } ; \widetilde{J}}=2-2 g_{\widetilde{J}}-V_{J}+L_{J} .
$$

$F_{\text {int } ; \widetilde{J}}$ can be further divided into $F_{\text {int; } \widetilde{J} ; \mathcal{G}}$, the number closed faces belonging to $\mathcal{G}$ and $F_{\text {int } ; \tilde{J} ; \mathcal{G}_{\text {color }}}$, the number of closed faces belonging to $\mathcal{G}_{\text {color }}$ and not to $\mathcal{G}$ :

$$
F_{\text {int } ; \widetilde{J}}=F_{\text {int } ; \widetilde{J} ; \mathcal{G}}+F_{\text {int } ; \widetilde{J} ; \mathcal{G}_{\text {color }}} .
$$

Summing over all jackets, we have

$$
\sum_{J}\left(F_{\text {int } ; \widetilde{J} ; \mathcal{G}}+F_{\text {int } ; \widetilde{J} ; \mathcal{G}_{\text {color }}}+F_{\text {ext } ; \widetilde{J}}\right)=2 F_{\text {int } ; \mathcal{G}}+2 F_{\text {int } ; \mathcal{G}_{\text {color }}}+\sum_{J} F_{\text {ext } ; \widetilde{J}}
$$

where, we recall that $F_{\text {int } ; \mathcal{G}_{\text {color }}}$ is the number of faces issued from $\mathcal{G}_{\text {color }}$ and not appearing in $\mathcal{G}$ and $F_{\text {int; } \mathcal{G}}$ is the number of closed faces of $\mathcal{G}$. 
The quantity $F_{\text {int } ; \mathcal{G}_{\text {color }}}$ can be computed explicitly: each $\varphi^{4}$ vertex contains 4 internal faces coming from the coloring (and not present in $\mathcal{G}$ ) and each $\varphi^{2}$ type vertex contains 3 of them. Then, one has

$$
F_{\text {int } ; \mathcal{G}_{\text {color }}}=4 V_{4}+3\left(V_{2}+\sum_{s=1}^{3} V_{s ; 2}^{\prime}\right) .
$$

Besides, using (28), we have

$$
\sum_{J}\left(-V_{J}+L_{J}\right)=3\left(V_{\mathcal{G}_{\text {color }}}-\frac{1}{2} N_{\text {ext }}\right)=3\left(4 V_{4}+2\left(V_{2}+\sum_{s=1}^{3} V_{s ; 2}^{\prime}\right)-\frac{1}{2} N_{\text {ext }}\right) .
$$

Summing over $J$ in (31), and using the above relation, we get from (33) and (34)

$$
F_{\text {int } ; \mathcal{G}}=2 V_{4}-\frac{3}{4} N_{\text {ext }}+3-\sum_{J} g_{\widetilde{J}}-\frac{1}{2} \sum_{J} F_{\text {ext } ; \widetilde{J}} .
$$

We re-express $\sum_{J} F_{\text {ext: } \widetilde{J}}$ in terms of topological numbers of the boundary graph $\partial \mathcal{G}$. The boundary $\partial \mathcal{G}$ is defined from its number of vertices $V_{\partial \mathcal{G}}$ and lines $L_{\partial \mathcal{G}}$ such that

$$
V_{\partial \mathcal{G}}=N_{\text {ext }}, \quad L_{\partial \mathcal{G}}=F_{\text {ext }} .
$$

The external legs of the initial graph $\mathcal{G}$ have 3 strands and we have

$$
3 N_{\text {ext }}=2 F_{\text {ext }} .
$$

The boundary graph is a closed ribbon graph, thus, $\partial \mathcal{G}$ has a single and closed jacket, itself. Note that a boundary graph may have several connected components. We get from the Euler formula

$$
2 C_{\partial \mathcal{G}}-2 g_{\partial \mathcal{G}}=V_{\partial \mathcal{G}}-L_{\partial \mathcal{G}}+F_{\partial \mathcal{G}},
$$

where $F_{\partial \mathcal{G}}$ and $C_{\partial \mathcal{G}}$ are, respectively, the number of faces and of connected components of $\partial \mathcal{G}$. It is simple to deduce from (37) and (38) that $L_{\partial \mathcal{G}}-V_{\partial \mathcal{G}}=N_{\text {ext }} / 2$ and, from the latter and the above Euler formula, one recovers

$$
F_{\partial \mathcal{G}}=2\left(C_{\partial \mathcal{G}}-1\right)-2 g_{\partial \mathcal{G}}+2+\frac{1}{2} N_{\text {ext }} .
$$

We remark that

$$
\sum_{J} F_{\mathrm{ext} ; \widetilde{J}}=F_{\partial \mathcal{G}}
$$

because each face of the boundary graph is uniquely represented in a unique $\widetilde{J}$. Indeed, we recall that a face of the boundary $\partial \mathcal{G}$ can be represented by a color triple $(0 a b)$; a face $(0 a b)$ belongs to a jacket $\widetilde{J}$ if $\widetilde{J}$ is the form $(0 a c b)$. A jacket being a cycle this implies that $c$ should be fixed (See Fig.7). Then evaluating (41) via (40), and inserting the result in (36), it can be inferred

$$
F_{\text {int } ; \mathcal{G}}=2 V_{4}-\frac{3}{4} N_{\text {ext }}+3-\sum_{J} g_{\widetilde{J}}-\frac{1}{2}\left(2\left(C_{\partial \mathcal{G}}-1\right)-2 g_{\partial \mathcal{G}}+2+\frac{1}{2} N_{\text {ext }}\right)
$$



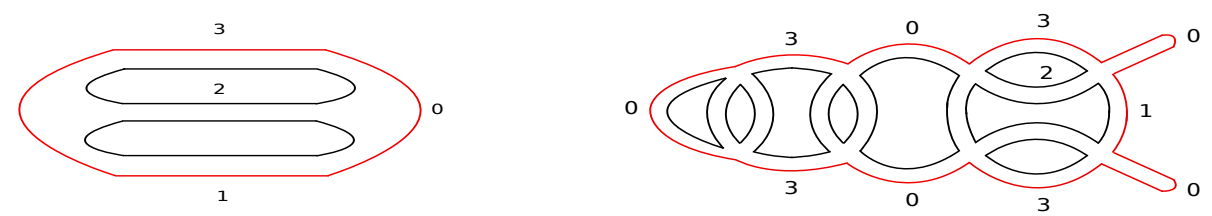

Figure 7: A face (highlighted) of $\partial \mathcal{G}$ (boundary graph of $\mathcal{G}$ Fig, 4) labeled by colors (013) and the unique pinched jacket $\widetilde{J}(0123)$ which possesses this face.

$$
=2 V_{4}-N_{\text {ext }}+2-\sum_{J} g_{\widetilde{J}}-\left(C_{\partial \mathcal{G}}-1\right)+g_{\partial \mathcal{G}}
$$

Inserting the latter in $\omega_{d}(\mathcal{G})=-L+F_{\text {int } ; \mathcal{G}}+V_{2}^{\prime}$, where $L=(1 / 2)\left[4 V_{4}+2\left(V_{2}+\sum_{s=1}^{3} V_{s ; 2}^{\prime}\right)-\right.$ $N_{\text {ext }}$, we finally get (27) which achieves the proof of the theorem.

The quantity

$$
-\sum_{J} g_{\widetilde{J}}+g_{\partial \mathcal{G}}-\left(C_{\partial \mathcal{G}}-1\right)
$$

should be analyzed in detail since, mainly, the classification of divergent graphs relies on its behavior. The next section is devoted to this study.

\subsection{Bounds on genera}

This section undertakes the study of the quantity $-\sum_{J} g_{\widetilde{J}}+g_{\partial \mathcal{G}}$ which turns out to be the central object capturing the behavior of the divergence degree. From that result, we will be able to classify the primitively divergent graphs in a next stage.

The strategy here follows mainly the same adopted in [45]: we perform a sequence of $0 k$-dipole contraction [36, 55, 56] of a given graph and scrutinize the genus change under such moves.

Let us give a flavor of the following combinatorial analysis before starting it. A $k$-dipole contraction on a colored vacuum graph [36] generalizes to the tensor case, the so-called line contractions along a tree for matrix ribbon graphs. Performing such a sequence erases all bubbles with melonic structure and one gets another graph which can be called a Filk rosette [57] of the tensor kind. The degree of that graph $\omega(\mathcal{G})=\sum_{J} g_{J}$ is the same as the degree of the initial graph since the genus of each jacket along this sequence of contractions is preserved.

In the present case (and also in [45]), starting from a colored theory with colored graphs, we do not perform arbitrary $k$-dipole contractions but, at first, $k$-dipole contractions involving all colors but 0 . The result is a graph with the same degree as previously claimed. In particular, erasing all melonic contributions yields exactly a graph of our starting theory. In a second step, we start what we call a $0 k$-dipole contraction involving now the last color 0 (a precise definition will be given below). Under such a contraction, the graph may or may not change of degree. We should analyze in detail the consequence of performing the contraction. The main points revealed in [45] were: (1) the boundary graph coincides with the resulting graph obtained after any full $0 k$-dipole contraction in an arbitrary sequence 


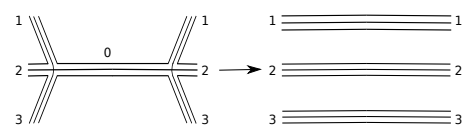

00-dipole

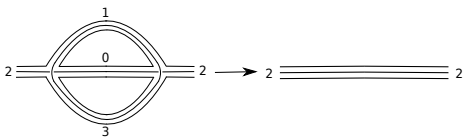

02-dipole

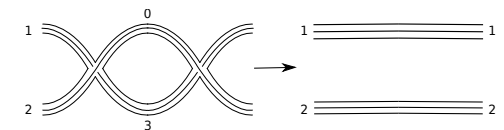

01-dipole

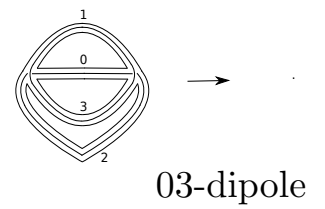

Figure 8: $0 k$-dipole contractions.

and removal of external structure of a given graph; (2) under a single $0 k$-dipole contraction, the genus of a jacket never increases and (3) the sum over all jackets of differences between genera of these jackets before and after contraction is always bounded from below by a fixed constant.

In the present context, we actually expect the same properties for graphs.

Definition 2 (0k-dipole and contraction). We define a $0 k$-dipole, $k=0,1,2,3$, as a maximal subgraph of $\mathcal{G}_{\text {color }}$ made of $k+1$ lines joining two vertices, one of which of color 0 . Maximal means the $0 k$-dipole is not included in a $0(k+1)$-dipole.

The contraction of a $0 k$-dipole erases the $k+1$ lines of the dipole and joins the remaining $3-k$ lines on both sides of the dipole by respecting colors (see Fig.8).

We summarize in the following proposition a basic fact about the boundary graph (the proof is totally similar of that of Lemma 3(Graph Contraction) in [45])

Proposition 1 (Graph contraction). Performing the maximal number $\left(4 V_{4}-N_{\text {ext }}\right) / 2$ of $0 k$-dipole contractions on $\mathcal{G}_{\text {color }}$ in any arbitrary order and erasing the external legs and the remaining open faces of $\mathcal{G}$ leads to the boundary graph $\partial \mathcal{G}$.

We restrict the analysis to the only significant situation of graphs without any two-points vertices of the kinds $V_{2}$ and $V_{s ; 2}^{\prime}{ }^{4}$ and aim at studying the change in genus of a single closed jacket under a dipole contraction. We write

$$
\mathcal{G}_{\text {color }} \rightarrow \mathcal{G}_{\text {color }}^{\prime}, \quad J \rightarrow J^{\prime}, \quad g_{\widetilde{J}} \rightarrow g_{\widetilde{J}^{\prime}}
$$

The analysis of the sum of differences between genera before and after the contraction will be a corollary of that result.

Consider a colored connected graph $\mathcal{G}_{\text {color }}$, a fixed $0 k$-dipole and the contracted graph $\mathcal{G}_{\text {color }}^{\prime}$, which may or may not be connected. After a contraction, the numbers of vertices and lines meet the following modifications:

$$
V \rightarrow V^{\prime}=V-2, \quad L \rightarrow L^{\prime}=L-4
$$

whereas the number of connected components may change from $c=1$ to $c^{\prime} \leq 3$.

\footnotetext{
${ }^{4}$ We can first analyze graphs for which one performs a full and maximal contraction of all $\varphi^{2}$ type vertices into a single line. After, the case including such vertices can be derived by re-inserting $\varphi^{2}$ vertex chains from that point.
} 
The other ingredient entering in the definition of genus is the number of faces. The change in faces can be handled by another notion of pair types associated with dipole contractions (see Appendix A.1). The key observations are summarized by the following statements:

Lemma 3 (Decreasing genera). Given a pinched jacket $\widetilde{J}$, we have

$$
g_{\widetilde{J}}-g_{\widetilde{J}^{\prime}} \geq 0
$$

Proof. See Appendix A.1.

Lemma 4 (Genus bounds). We have

$$
g_{\widetilde{J}} \geq g_{\partial \mathcal{G}}, \quad \sum_{J} g_{\widetilde{J}}-3 g_{\partial \mathcal{G}} \in \mathbb{N} .
$$

Proof. A complete sequence of $0 k$-dipole contractions on the graph $\mathcal{G}$ yields the graph $\partial \mathcal{G}$ (see Proposition 1). Using Lemma 3, any genus of any pinched jacket $\widetilde{J}$ decreases along that sequence of contractions. Finally, the pinched jacket coincides with the boundary graph ${ }^{5}$ itself $\partial \mathcal{G}$, which proves (47). The factor of 3 appearing in the second statement of (47), come from the fact that there must exist at least three jackets $\widetilde{J}$ (in the sequence) generating the boundary graph which possess a greater genus. Indeed, if $(a b c)$ defines the boundary graph cycle (obtained after contracting the color 0 , such that $a, b, c \neq 0)$, then three jackets $(0 a b c)$, $(a 0 b c)$ and $(a b 0 c)$ contract back on $(a b c)$. Some of the "ancestors" (placed upstream with respect to the sequence of contractions) of these three jackets or the three jackets themselves should possess a genus strictly greater than $g_{\partial \mathcal{G}}$.

\subsection{Divergent graphs}

We always assume that $\partial \mathcal{G} \neq \emptyset$ hence $C_{\partial \mathcal{G}} \geq 1$. Furthermore $C_{\partial \mathcal{G}} \leq N_{\text {ext }} / 2$, because each connected component must have at least a non zero even number of external legs.

After an analysis (the details of which are collected in Appendix A.2), the following table gives a list of primitively divergent graphs:

\begin{tabular}{lcccc||c}
$N_{\text {ext }}$ & $V_{2}$ & $g_{\partial \mathcal{G}}$ & $C_{\partial \mathcal{G}}-1$ & $\sum_{\widetilde{J}} g_{\widetilde{J}}$ & $\omega_{d}(\mathcal{G})$ \\
\hline \hline 4 & 0 & 0 & 0 & 0 & 0 \\
\hline 2 & 0 & 0 & 0 & 0 & 1 \\
2 & 1 & 0 & 0 & 0 & 0 \\
2 & 0 & 0 & 0 & 1 & 0 \\
\hline \hline
\end{tabular}

Table 1: List of primitively divergent graphs

We emphasize the fact that the anomalous term of the form $\left(\int \phi^{2}\right)\left(\int \phi^{2}\right)$ occurring in four dimensions [45] does not appear here. One could claims that in higher dimensions, more anomalous might be present. From the above table characterizing all dangerous contributions, we are in position to address the renormalization program for these amplitudes and this is the purpose of the next section.

${ }^{5}$ This results from Proposition 1 ; since after a full sequence of contractions $\mathcal{G} \rightarrow \partial \mathcal{G}$, any pinched jacket of $\mathcal{G}$ should have a projected in $\partial \mathcal{G}$. However $\partial \mathcal{G}$ is unique so that the conclusion is immediate. 


\section{Renormalization}

The renormalization procedure of primitively divergent graphs could be implement in the way of [45] if one switches to the direct space using techniques developed in [52, 50]. We equivalently perform the renormalization procedure in the momentum space in a similar way of [51].

The renormalization schemes corresponding to any primitively divergent contribution given by Table 1 have to be studied. Nevertheless, the procedure remaining similar at a given number of external legs, we will only treat the following cases:

(i) $N_{\text {ext }}=4$ yielding $\omega_{d}(\mathcal{G})=0$ (first line of Table 1 );

(ii) $N_{\text {ext }}=2$ yielding $\omega_{d}(\mathcal{G})=1$ (second line of Table 1 ).

We emphasize that even though the above cases (and for the first situation, for a given vertex configuration) will be discussed, the analysis performed in the following can be carried out for all possible cases and affords the same conclusion.

\subsection{Renormalization of the four-point function}

Consider a four-point function subgraph $G_{i}^{k}$ characterized by the first line of Table 1 and equipped with four external propagators. The graph is such that $g_{\partial \mathcal{G}}=0$, hence has a boundary graph of the melonic type. The pattern followed by external momenta are necessarily of the form $V_{4}$ (see Fig, 2). Denoting external momenta data by $p_{f}^{\text {ext }}, f=1,2,3,1^{\prime}, 2^{\prime}, 3^{\prime}$, we assign to each $p_{f}^{\text {ext }}$ an external or boundary face (there are six of them). Calculations will be only made for the initial configuration of the $V_{4}$ vertex given by (8). It will be obvious that for any other permutation, the derivations will lead to similar conclusions.

Let $\mathcal{A}_{4}\left(G_{i}^{k}\right)$ be the amplitude of the resulting graph. Note that the external leg indices are at scale $j_{l}$ which must be strictly smaller than $i$ the scale index of the quasi-local subgraph $G_{i}^{k}$.

Using the face factorization, $\mathcal{A}_{4}\left(G_{i}^{k}\right)$ can be written (alleviating notations, henceforth $p_{s}$ refers directly to the absolute value $\left|p_{s}\right|$ and the reference graph $G_{i}^{k}$ will be dropped) as

$$
\mathcal{A}_{4}\left[\left\{p_{f}^{\mathrm{ext}}\right\}\right]=\sum_{p_{f}} \int\left[\prod_{\ell} d \alpha_{\ell} e^{-\alpha_{\ell} m}\right]\left\{\left[\prod_{f \in F_{\mathrm{ext}}} e^{-\left(\sum_{\ell \in f} \alpha_{\ell}\right) a_{f} p_{f}^{\mathrm{ext}}}\right]\left[\prod_{f \in F_{\mathrm{int}}} e^{-\left(\sum_{\ell \in f} \alpha_{\ell}\right) a_{f} p_{f}}\right]\right\} .
$$

each $\alpha_{\ell} \in\left[M^{-d_{\ell}}, M^{-d_{\ell}+1}\right], \ell$ runs over all lines of the graph. Referring to an external line, we will use instead $l$. Hence, $d_{\ell}$ can be either an external index $j_{l}$ or an internal one $i_{\ell}$. In particular, for all $f \in F_{\text {int }}$ in the second product, $\alpha_{\ell}$ is at scale $i_{\ell} \gg j_{l}$.

Next, we single out for each independent external face the exponent $\alpha_{l} p_{f}^{\text {ext }}$, where $\alpha_{l} \in$ $\left[M^{-j_{l}}, M^{-j_{l}+1}\right]$, such that, we can write a given external face amplitude as

$$
e^{-\left(\sum_{\ell \in f} \alpha_{\ell}\right) a_{f} p_{f}^{\text {ext }}}=e^{-\left(\alpha_{l}+\alpha_{l^{\prime}}\right) a_{f} p_{f}^{\text {ext }}} e^{-\left(\sum_{\ell \in f / \ell \neq l} \alpha_{\ell}\right) a_{f} p_{f}^{\text {ext }}} .
$$

Noting that all $\alpha_{\ell \neq l}$, in the sum, are now at scale $i_{\ell} \gg j_{l}$, and $p_{f}^{\text {ext }} \sim M^{j_{l}}$, we perform a Taylor expansion for each external face as

$$
e^{-\left(\sum_{\ell \in f} \alpha_{\ell}\right) a_{f} p_{f}^{\mathrm{ext}}}=e^{-\left(\alpha_{l}+\alpha_{l^{\prime}}\right) a_{f} p_{f}^{\mathrm{ext}}}\left[1-\left(\sum_{\ell \in f / \ell \neq l} \alpha_{\ell}\right) a_{f} p_{f}^{\mathrm{ext}} \int_{0}^{1} d t e^{-t\left(\sum_{\ell \in f / \ell \neq l} \alpha_{\ell}\right) a_{f} p_{f}^{\mathrm{ext}}}\right] .
$$


Rewriting (50) as $e^{-\left(\sum_{\ell \in f} \alpha_{\ell}\right) a_{f} p_{f}^{\text {ext }}}=e^{-\left(\alpha_{l}+\alpha_{l^{\prime}}\right) a_{f} p_{f}^{\text {pxt }}}\left[1-R_{f}^{\text {ext }}\right]$, where $R_{f}^{\text {ext }}$ is the remainder of the Taylor expansion, we substitute the latter expression in the initial amplitude $\mathcal{A}_{4}\left[\left\{p_{f}^{\text {ext }}\right\}\right]$. In loosely notations, the result is

$$
\begin{aligned}
& \mathcal{A}_{4}\left[\left\{p_{f}^{\mathrm{ext}}\right\}\right]=\sum_{p_{f}} \int\left[\prod_{\ell} d \alpha_{\ell} e^{-\alpha_{\ell} m}\right]\{ \\
& \left.\left[\prod_{f \in F_{\text {ext }}} e^{-\left(\alpha_{l}+\alpha_{l^{\prime}}\right) a_{f} p_{f}^{\text {ext }}}\right]\left[1-\sum_{f \in F_{\text {ext }}} R_{f}^{\text {ext }}+\sum_{f, f^{\prime} \in F_{\text {ext }}} R_{f}^{\text {ext }} R_{f^{\prime}}^{\text {ext }}-\ldots\right]\left[\prod_{f \in F_{\text {int }}} e^{-\left(\sum_{\ell \in f} \alpha_{\ell}\right) a_{f} p_{f}}\right]\right\} .
\end{aligned}
$$

Collecting the leading order contribution, one has

$$
\begin{aligned}
& \mathcal{A}_{4}\left[\left\{p_{f}^{\mathrm{ext}}\right\} ; 0\right]=\sum_{p_{f}} \int\left[\prod_{\ell} d \alpha_{\ell} e^{-\alpha_{\ell} m}\right]\left\{\left[\prod_{f \in F_{\mathrm{ext}}} e^{-\left(\alpha_{l}+\alpha_{l^{\prime}}\right) a_{f} p_{f}^{\mathrm{ext}}}\right]\left[\prod_{f \in F_{\text {int }}} e^{-\left(\sum_{\ell \in f} \alpha_{\ell}\right) a_{f} p_{f}}\right]\right\}(52) \\
& =\left\{\int\left[\prod_{l} d \alpha_{l} e^{-\alpha_{l} m}\right] \prod_{f \in F_{\mathrm{ext}}} e^{-\left(\alpha_{l}+\alpha_{l^{\prime}}\right) a_{f} p_{f}^{\mathrm{ext}}}\right\}\left\{\sum_{p_{f}} \int\left[\prod_{\ell \neq l} d \alpha_{\ell} e^{-\alpha_{\ell} m}\right] \prod_{f \in F_{\text {int }}} e^{-\left(\sum_{\ell \in f} \alpha_{\ell}\right) a_{f} p_{f}}\right\} .
\end{aligned}
$$

The first factor corresponds to a vertex hooked to four external propagators since it can be recast in a mere form

$$
\begin{aligned}
& \int\left[\prod_{l} d \alpha_{l} e^{-\alpha_{l} m}\right]\left[\prod_{f \in F_{\mathrm{ext}}} e^{-\left(\alpha_{l}+\alpha_{l^{\prime}}\right) a_{f} p_{f}^{\text {ext }}}\right] \\
& =\int\left[\prod_{l} d \alpha_{l}\right]\left\{e^{-\alpha_{l_{1}}\left(a_{1} p_{1}+a_{2} p_{2}+a_{3} p_{3}+m\right)} e^{-\alpha_{l_{2}}\left(a_{1} p_{1}^{\prime}+a_{2} p_{2}+a_{3} p_{3}+m\right)}\right. \\
& \left.e^{-\alpha_{l_{3}}\left(a_{1} p_{1}^{\prime}+a_{2} p_{2}^{\prime}+a_{3} p_{3}^{\prime}+m\right)} e^{-\alpha_{l_{4}}\left(a_{1} p_{1}+a_{2} p_{2}^{\prime}+a_{3} p_{3}^{\prime}+m\right)}\right\} .
\end{aligned}
$$

The second factor coincides with the logarithmically divergent internal contribution given by the power-counting theorem. Hence $\mathcal{A}_{4}\left[\left\{p_{f}^{\text {ext }}\right\} ; 0\right]$ determines a log-divergent counterterm participating in the vertex renormalization.

The remaining terms in (51) should be analyzed. Proving that the first subleading order contribution improves the power-counting will be a sufficient condition to ensure the convergence of the subsequent product terms. The first subleading term is of the form

$$
\begin{aligned}
& R_{4}=\sum_{p_{f}} \int\left[\prod_{\ell} d \alpha_{\ell} e^{-\alpha_{\ell} m}\right]\left\{\left[\prod_{f \in F_{\mathrm{ext}}} e^{-\left(\alpha_{l}+\alpha_{l^{\prime}}\right) a_{f} p_{f}^{\mathrm{ext}}}\right]\right. \\
& \left.\times\left[-\sum_{f \in F_{\mathrm{ext}}}\left(\sum_{\ell \in f / \ell \neq l} \alpha_{\ell}\right) a_{f} p_{f}^{\text {ext }} \int_{0}^{1} d t e^{-t\left(\sum_{\ell \in f / \ell \neq l} \alpha_{\ell}\right) a_{f} p_{f}^{\text {ext }}}\right]\left[\prod_{f \in F_{\text {int }}} e^{-\left(\sum_{\ell \in f} \alpha_{\ell}\right) a_{f} p_{f}}\right]\right\}
\end{aligned}
$$

and can be bounded by

$$
\left|R_{4}\right| \leq K M^{-\left(i\left(G_{i}^{k}\right)-e\left(G_{i}^{k}\right)\right)} \sum_{p_{f}} \int\left[\prod_{\ell} d \alpha_{\ell} e^{-\alpha_{\ell} m}\right]\left[\prod_{f \in F_{\text {int }}} e^{-\left(\sum_{\ell \in f} \alpha_{\ell}\right) a_{f} p_{f}}\right],
$$

where $K$ is some constant (which could depend on the number of internal lines of the graph and number of external faces). Note that the integral over $t$ yields just a factor of $O(1)$. Also 
remark that, we used optimal bounds such that $p_{f}^{\text {ext }} \leq M^{e\left(G_{i}^{k}\right)}$, and, for any internal decay, one assumes that $\alpha_{\ell} \leq M^{-i\left(G_{i}^{k}\right)}$, recalling that $e\left(G_{i}^{k}\right)=\sup _{l} j_{l}$ and $i\left(G_{i}^{k}\right)=\inf _{\ell \in G_{i}^{k}} i_{\ell}$. The factor $M^{-\left(i\left(G_{i}^{k}\right)-e\left(G_{i}^{k}\right)\right)}$ guarantees that the power-counting $M^{\omega_{d}\left(G_{i}^{k}\right)=0}$ (which corresponds to the last sum, up to some constant) is improved and will bring the sufficient decay to ensure the sum of momentum attributions in the standard way of [52].

\subsection{Renormalization of the two-point function}

We consider a graph $G_{i}^{k}$ with two external legs with topology as dictated by one the corresponding rows of Table 1 . Note that the boundary data $p_{f}^{\text {ext }}, f=1,2,3$, now follow the pattern of a simple mass vertex. Only, the case of a two-point graph with a linear divergence will be treated since the subtraction of log-divergent cases can be recovered easily from the same analysis by restricting the Taylor expansion at less order.

Let $\mathcal{A}_{2}\left[\left\{p_{f}^{\text {ext }}\right\}\right]$ be the full amplitude associated with the graph $G_{i}^{k}$ with external propagators. Having defined all tools in the previous analysis of Subsection 5.1, we perform the following expansion of any boundary face amplitude occurring in $\mathcal{A}_{2}\left[\left\{p_{f}^{\text {ext }}\right\}\right]$ as

$$
\begin{aligned}
& e^{-\left(\sum_{\ell \in f} \alpha_{\ell}\right) a_{f} p_{f}^{\mathrm{ext}}}=e^{-\left(\alpha_{l}+\alpha_{l^{\prime}}\right) a_{f} p_{f}^{\mathrm{ext}}} \\
& \times\left[1-\left(\sum_{\ell \in f / \ell \neq l} \alpha_{\ell}\right) a_{f} p_{f}^{\mathrm{ext}}+\left[-\left(\sum_{\ell \in f / \ell \neq l} \alpha_{\ell}\right) a_{f} p_{f}^{\mathrm{ext}}\right]^{2} \int_{0}^{1} d t(1-t) e^{-t\left(\sum_{\ell \in f / \ell \neq l} \alpha_{\ell}\right) a_{f} p_{f}^{\mathrm{ext}}}\right] .
\end{aligned}
$$

The same 56 can rewritten as $e^{-\left(\sum_{\ell \in f} \alpha_{\ell}\right) a_{f} p_{f}^{\text {ext }}}=e^{-\left(\alpha_{l}+\alpha_{l^{\prime}}\right) a_{f} p_{f}^{\text {ext }}}\left[1+S_{f}^{\text {ext }}+R_{f}^{\text {ext }}\right]$. Substituting this expression in the initial amplitude yields

$$
\begin{aligned}
& \mathcal{A}_{2}\left[\left\{p_{f}^{\mathrm{ext}}\right\}\right]=\sum_{p_{f}} \int\left[\prod_{\ell} d \alpha_{\ell} e^{-\alpha_{\ell} m}\right]\{ \\
& {\left[\prod_{f \in F_{\mathrm{ext}}} e^{-\left(\alpha_{l}+\alpha_{l^{\prime}}\right) a_{f} p_{f}^{\mathrm{ext}}}\right]\left[1+\sum_{f \in F_{\mathrm{ext}}} S_{f}^{\mathrm{ext}}+\sum_{f \in F_{\mathrm{ext}}} R_{f}^{\mathrm{ext}}\right.} \\
& \left.+\sum_{f, f^{\prime} \in F_{\mathrm{ext}}}\left(S_{f}^{\mathrm{ext}}+R_{f}^{\mathrm{ext}}\right)\left(S_{f^{\prime}}^{\mathrm{ext}}+R_{f^{\prime}}^{\mathrm{ext}}\right)+\ldots\right]\left[\prod_{f \in F_{\mathrm{int}}} e^{\left.-\left(\sum_{\ell \in f} \alpha_{\ell}\right) a_{f} p_{f}\right]} .\right.
\end{aligned}
$$

As expected, the leading order contribution $\mathcal{A}_{2}\left[\left\{p_{f}^{\text {ext }}\right\} ; 0\right]$ is mainly of the factorized form 52 with, in the present instance, the first factor appearing as

$$
\begin{aligned}
& \int\left[\prod_{l} d \alpha_{l} e^{-\alpha_{l} m}\right]\left[\prod_{f \in F_{\mathrm{ext}}} e^{-\left(\alpha_{l}+\alpha_{l^{\prime}}\right) a_{f} p_{f}^{\mathrm{ext}}}\right] \\
& =\int\left[\prod_{l} d \alpha_{l}\right] e^{-\alpha_{l_{1}}\left(a_{1} p_{1}+a_{2} p_{2}+a_{3} p_{3}+m\right)} e^{-\alpha_{l_{2}}\left(a_{1} p_{1}+a_{2} p_{2}+a_{3} p_{3}+m\right)}
\end{aligned}
$$

and the second factor bringing a linear divergence. Clearly, this term belongs to a mass renormalization.

The remaining task is to prove that higher order terms improve the power-counting. Let us focus on the following

$$
\mathcal{A}_{2}^{\prime}\left[\left\{p_{f}^{\text {ext }}\right\} ; 0\right]=\sum_{p_{f}} \int\left[\prod_{\ell} d \alpha_{\ell} e^{-\alpha_{\ell} m}\right]\{
$$




$$
\left.\left[\prod_{f \in F_{\mathrm{ext}}} e^{-\left(\alpha_{l}+\alpha_{l^{\prime}}\right) a_{f} p_{f}^{\mathrm{ext}}}\right]\left[-\sum_{f \in F_{\mathrm{ext}}}\left(\sum_{\ell \in f / \ell \neq l} \alpha_{\ell}\right) a_{f} p_{f}^{\mathrm{ext}}\right]\left[\prod_{f \in F_{\mathrm{int}}} e^{-\left(\sum_{\ell \in f} \alpha_{\ell}\right) a_{f} p_{f}}\right]\right\}
$$

which can be seen as a wave-function renormalization. Indeed, we can recompose it as

$$
\begin{aligned}
& \mathcal{A}_{2}^{\prime}\left[\left\{p_{f}^{\mathrm{ext}}\right\} ; 0\right]=-\sum_{s=1}^{3}\{ \\
& {\left[\int\left[\prod_{l} d \alpha_{l}\right] e^{-\alpha_{l_{1}}\left(a_{1} p_{1}+a_{2} p_{2}+a_{3} p_{3}+m\right)} e^{-\alpha_{l_{2}}\left(a_{1} p_{1}+a_{2} p_{2}+a_{3} p_{3}+m\right)} a_{s} p_{s}^{\mathrm{ext}}\right]} \\
& \left.\left[\sum_{p_{f}} \int\left[\prod_{\ell \neq l} d \alpha_{\ell} e^{-\alpha_{\ell} m}\right]\left(\sum_{\ell \in f_{s} / \ell \neq l} \alpha_{\ell}\right) \prod_{f \in F_{\mathrm{int}}} e^{-\left(\sum_{\ell \in f} \alpha_{\ell}\right) a_{f} p_{f}}\right]\right\},
\end{aligned}
$$

where the first factor is nothing but a wave function type contribution (and renormalizes $a_{s}$ ) and the second factor is log-divergent. The latter statement is justified by the fact that the factors $\sum_{\ell \in f_{s} / \ell \neq l} \alpha_{\ell}$ brought by the derivatives are of order $M^{-i_{\ell}}$ which annihilates the internal divergence degree which was linear.

As another consequence of the decay of $\sum_{\ell \in f / \ell \neq l} \alpha_{\ell}$, any monomial in $S_{f}$ of order higher than 2 in (57) is simply convergent.

Next, the first order remainder term is of the form

$$
\begin{aligned}
& R_{2}=\sum_{p_{f}} \int\left[\prod_{\ell} d \alpha_{\ell} e^{-\alpha_{\ell} m}\right]\left\{\left[\prod_{f \in F_{\mathrm{ext}}} e^{-\left(\alpha_{l}+\alpha_{l^{\prime}}\right) a_{f} p_{f}^{\mathrm{ext}}}\right]\right. \\
& \left.\sum_{f \in F_{\mathrm{ext}}}\left[\left[\left(\sum_{\ell \in f / \ell \neq l} \alpha_{\ell}\right) a_{f} p_{f}^{\mathrm{ext}}\right]^{2} \int_{0}^{1} d t(1-t) e^{-t\left(\sum_{\ell \in f / \ell \neq l} \alpha_{\ell}\right) a_{f} p_{f}^{\mathrm{ext}}}\right]\left[\prod_{f \in F_{\mathrm{int}}} e^{-\left(\sum_{\ell \in f} \alpha_{\ell}\right) a_{f} p_{f}}\right]\right\} .
\end{aligned}
$$

Using the fact that $p_{f}^{\text {ext }} \leq M^{-e\left(G_{i}^{k}\right)}$ whereas $\alpha_{\ell} \sim M^{-i_{\ell}}$, with $i_{\ell} \gg e\left(G_{i}^{k}\right)$, for $\ell \neq l$, we find the following optimal bound for the above term as

$$
\left|R_{2}\right| \leq K M^{-2\left(i\left(G_{i}^{k}\right)-e\left(G_{i}^{k}\right)\right)} \sum_{p_{f}} \int\left[\prod_{\ell \neq l} d \alpha_{\ell} e^{-\alpha_{\ell} m}\right]\left[\prod_{f \in F_{\mathrm{int}}} e^{-\left(\sum_{\ell \in f} \alpha_{\ell}\right) a_{f} p_{f}}\right],
$$

where the integral over $t$ yields again a factor of $O(1)$. We recognize, in the last sum, the linear divergence associated with the two-point graph at $t=0$. Hence, this term is convergent and will ensure the summability of scale attributions.

All the rest of terms are manifestly convergent from the fact that they involve only product of convergent terms. Finally, the summability of the scale attributions can be performed in the way introduced in [52].

\subsection{About enlarging and reducing the space of couplings}

It is worthwhile to discuss how the (perturbative) renormalizability of the initial model can be extended to different models with various degrees of anisotropy between colors. Different classes of models are obtained by just putting a different coupling for each term or conversely identifying the different couplings. 
Renormalizability is triggered by the coherent combination of three ingredients: a multiscale analysis, a locality principle and a power-counting theorem. The issue raised above can be rephrased as "is a given class of theory with particular anisotropies stable or not under the RG flow?".

Consider the renormalizable model defined by the action written in terms of (4) and (8), which we call in the following discussion (I). The model has many wave function couplings, namely $a_{s}$, and one single interaction coupling, $\lambda$ (the mass is always kept fixed in the next developments).

In addition to the model (I), let us discuss three other basic classes of models:

(1) The most general "anisotropic" model which has different couplings for each interaction and different wave function couplings; hence, in this model, the main parameters are $\left(\lambda_{\epsilon=1,2,3}, a_{\epsilon=1,2,3}\right)$;

(2) The model with "anisotropic interactions" which has different interaction couplings but a single wave function coupling $\left(\lambda_{\epsilon=1,2,3}, a_{\epsilon=1,2,3}=a\right)$;

(3) The "isotropic" model which has a single coupling for interactions and a single coupling for kinetic terms $\left(\lambda_{\epsilon=1,2,3}=\lambda, a_{\epsilon=1,2,3}=a\right)$.

Note that all these theories are continuously connected by parameter deformations. However, the interesting question is whether these classes are stable under the renormalization group flow.

All models (1)-(3) share a common feature with the model (I) studied so far: their multiscale analysis and power-counting theorem are identical since those are written without the explicit knowledge of the coupling constants. All what is required to perform a powercounting is the type of propagator decay, the vertices and lines combinatorial properties and gluing rules and these are exactly the same for these models. One can call these theories "power-counting renormalizable" to this respect.

However, as previously emphasized, the renormalizability is also about a locality principle that ought to be satisfied by the model. This feature can be also called "replicability" or "surviving property" along scales of the model. The locality principle in a just renormalizable model with marginal terms with $n$-valent vertices ensures, roughly, that any log-divergent $n$ point function with external data of the same form of a given interaction should renormalize the coupling of this interaction. This principle holds immediately either for a unique coupling or different ones for marginal terms. Indeed, one has just to decide, in any situation, to affect to the renormalized coupling constant of a given vertex all divergent terms with akin external data. Even for a unique coupling shared by several interactions, this can be explicitly done. Of course, from the point of view of the RG flow, the RG equations can be very involved (the following analysis performed at one-loop does not displays this feature but it certainly occurs are further loops) and each renormalized coupling is generally dependent of many (if not all) couplings. The key point is that they can be, at least, written in an unambiguous way, for one or many coupling constants for the interaction.

The actual and only issue during the renormalization occurs potentially with the locality principle for relevant terms. In the present story, relevant terms are two-point function $5^{6}$ given by the second line of Table 1 . Note that the second and third lines consist in marginal two-point functions and so can be simply handled by the mass renormalization.

\footnotetext{
${ }^{6}$ In several other contexts, they are always the most diverging ones.
} 
Consider model (I) as starting point. Performing a Taylor expansion of two-point functions around their local part (in the way already introduced in Subsection 5.2) yields two important corrections: a 0-th order linearly divergent contribution which again contributes to the mass and a first order log-divergent contribution involving $a_{s} p_{s}^{\text {ext }}$. For each two-point function, one finds that the second kind of correction has two features: (A) it can be identified with a term present in the Lagrangian and (B) it should contribute to the wave function renormalization $Z_{s}$ of the model.

Note that, for theories (I) and (1) having many coupling constants $a_{\epsilon=1,2,3}$, there is no ambiguity to renormalize independently each of these couplings by the above log-contribution of any two-point function and, so, to define $Z_{s}$. This is totally independent of the presence of several coupling $\lambda_{\epsilon=1,2,3}$. It becomes immediate that (I) and (1) satisfy a locality principle for two-point functions and therefore are renormalizable.

Let us focus on model (3) assuming that $\lambda_{\epsilon}=\lambda$, and $a_{\epsilon}=a$. Condition (A) above is fulfilled since (60) can be reached in any case. However, due to the fact that we have a single wave function coupling, Condition (B) changes drastically: It should be replaced by $\left(B^{\prime}\right)$ : the local log-divergent contribution of the two-point function should define a unique wave function renormalization $Z$ independent of the strand index $s$. One notes from (60), that the log-divergent term actually involves the strand index $s$ (see the last line where $\sum_{\ell \in f_{s} / \ell \neq l} \alpha_{\ell}$ is integrated). This issue can be overcome by a permutation of the vertices present in the theory. The latter statement is understood in the sense that given a graph which is not symmetric in the strands, there always exists a set of graphs (with the same number of vertices, lines and the same degree of divergence) identical by color permutation to the former. This simply holds by permutation the vertex indices $V_{4 ; 1} \rightarrow V_{4 ; 2} \rightarrow V_{4 ; 3}$. Hence, if the next order correction of a two-point graph has an amplitude $\mathcal{A}_{2}^{\prime}(60)$ depending on $s=1$, the same correction has two partners of the same form for $s=2$, 3 . Adding these restores the symmetry in $s$ and entails that the self-energy $\Sigma\left[p_{1}^{\text {ext }}, p_{2}^{\text {ext }}, p_{3}^{\text {ext }}\right]$ (the sum of all amputated 1PI two-point functions at external momenta $p_{s}^{\text {ext }}$ ) should be a symmetric function in all strands. The wave function renormalization

$$
\begin{aligned}
Z & =1-\left.\frac{1}{a} \partial_{p_{1}^{\text {ext }}} \Sigma\left[p_{1}^{\text {ext }}, p_{2}^{\text {ext }}, p_{3}^{\text {ext }}\right]\right|_{p_{s^{\prime}}^{\text {ext }}=0}=1-\left.\frac{1}{a} \partial_{p_{2}^{\text {ext }}} \Sigma\left[p_{1}^{\text {ext }}, p_{2}^{\text {ext }}, p_{3}^{\text {ext }}\right]\right|_{p_{s^{\prime}}^{\text {ext }}=0} \\
& =1-\left.\frac{1}{a} \partial_{p_{3}^{\text {ext }}} \Sigma\left[p_{1}^{\text {ext }}, p_{2}^{\text {ext }}, p_{3}^{\text {ext }}\right]\right|_{p_{s^{\prime}}^{\text {ext }}=0}
\end{aligned}
$$

should not depend on $s$. By expanding $\Sigma\left[b_{1}^{\text {ext }}, b_{2}^{\text {ext }}, b_{3}^{\text {ext }}\right]$ order by order and even though the latter is symmetric, the fact that $Z$ keeps a fixed value for all derivatives $\partial_{b_{s}^{\text {ext }}}$ cannot be met unless all coupling coincide: $\lambda_{\epsilon}=\lambda$. This condition clearly holds for the model (3) and therefore the latter is renormalizable. In contrast, the model (2) having distinct $\lambda_{\epsilon}$ is not stable under RG flow: starting from a bare action in the class (2), one ends up with an effective action in the general class (1).

Let us discuss, finally, the following peculiar partly anisotropic theory

(4) Consider the model with two interaction couplings and two different wave function couplings such that $\lambda_{1} \neq \lambda_{2}=\lambda_{3}, a_{1} \neq a_{2}=a_{3}$; note that we could have chosen any symmetric situation.

The model (4) is interesting because, we do not choose the easy case to let all $a_{s}$ to be different (which will obviously lead to renormalizability along with theories (I) and (1)), but 
we choose instead two wave function couplings $a_{2}=a_{3} \neq a_{1}$. This model is renormalizable because it lies exactly in between the two different situations so far carried out.

Merging the sector $a_{2}=a_{3}$ and following step by step the above analysis, it is direct to find the necessary condition $\lambda_{2}=\lambda_{3}$ for having a unique wave function renormalization $Z_{2}=Z_{3}$. We conclude that the model (4) is renormalizable.

The model (4) is interesting by itself for the remaining analysis on $\beta$ function. Indeed, it is related to two renormalizable models which are, furthermore, asymptotically free (see next section):

(4') $\lambda_{1} \neq \lambda_{2}=\lambda_{3}=0, a_{1} \neq a_{2}=a_{3}$;

(4") $0=\lambda_{1} \neq \lambda_{2}=\lambda_{3}, a_{1} \neq a_{2}=a_{3}$.

It is not excluded that there exist other renormalizable theories in this framework. The above analysis illustrates the richness of tensor field theories.

\section{6 one-loop $\gamma$-, $\beta$-functions and RG flows}

This section starts the second part of our analysis which aims at calculating the so-called $\gamma$ - and $\beta$-functions governing coupling constant RG flows of the model. We will restrict the study at one-loop since it is well-known that important properties (like asymptotic freedom for instance) of the model can be inferred even at this approximation.

The $U(1)^{3}$ model described so far has a unique coupling constant associated with all vertices $V_{4}$. It is interesting to relax that condition and study the same model in full generality by assigning to each vertex a different coupling constant. According to the discussion of Subsection 5.3, the model obtained in this way is again renormalizable. The RG equations for the renormalizable model (3) (from Subsection 5.3) can be inferred from this extended model. Meanwhile, RG equations for models (4') and (4') have to be computed with slightly modified method. Moreover, we introduce a symmetry factor due to the internal symmetry of the vertices: each coupling constants will be of the form $-\lambda_{\epsilon} / 2, \epsilon=1,2,3$.

In the following, the basic quantities (the self-energy and $\Gamma^{4}$-function) are first computed in full generality (i.e. within the framework of model (1)) and, then, we will particularize these on three reduced theories given by

(i) $\left[a_{\epsilon}=a, \lambda_{\epsilon}=\lambda\right]$

(ii) $\left[a_{2}=a_{3} \neq a_{1}, \quad \lambda_{2,3}=0, \quad \lambda_{1} \neq 0\right]$

(iii) $\left[a_{2}=a_{3} \neq a_{1}, \quad \lambda_{2}=\lambda_{3} \neq 0, \quad \lambda_{1}=0\right]$

The instance (i) corresponds to an interesting model with a unique coupling constant for all interactions (8) and a unique wave function coupling. It is simply the most natural model which can be viewed as the rank-3 analogue of the model investigated in [45]. On the other hand, the second and third correspond to some more drastic truncation that one could perform. The latter (ii) and (iii) share some but not all features of the former hence are different. 


\section{$6.1 \gamma$ - and $\beta$-functions}

In full generality (working in the model (1) Subsection 5.3), considering three wave function renormalizations $Z_{\epsilon=1,2,3}$, each of one with respect to each of the strands, the field strength can be modified as follows:

$$
\varphi \longrightarrow\left(Z_{1} Z_{2} Z_{3}\right)^{\frac{1}{6}} \varphi
$$

so that, after renormalization, the wave function couplings satisfy the equations

$$
a_{\epsilon}^{\text {ren }}=a_{\epsilon}\left(\frac{Z_{\epsilon}^{2}}{Z_{\check{\epsilon}} Z_{\check{\epsilon}}}\right)^{\frac{1}{3}}, \quad \epsilon=1,2,3, \quad \check{\epsilon} \neq \epsilon, \quad \check{\epsilon} \neq \check{\check{\epsilon}} \neq \epsilon .
$$

For a rank three tensor model as the one we are presently dealing with, we define

$$
Z_{\epsilon}=1-\left.\frac{1}{a_{\epsilon}} \partial_{b_{\epsilon}} \Sigma\right|_{b_{1,2,3}=0}
$$

where the self-energy $\Sigma\left(b_{1}, b_{2}, b_{3}\right)$ is the sum of the amputated one particle irreducible (1PI) amplitudes of the two-point correlation function truncated at one-loop that we denote

$$
\Sigma\left(b_{1}, b_{2}, b_{3}\right)=\left\langle\bar{\varphi}_{b_{1} b_{2} b_{3}} \varphi_{b_{1} b_{2} b_{3}}\right\rangle_{1 P I}^{t}
$$

Our initial goal is to compute at one-loop the dynamics of the effective couplings $a_{\epsilon}$ governed by the $\gamma_{\epsilon}$-functions encoded in (65). In a second stage, we will compute, still at one-loop, the mass $\beta_{m}$-function given by the expression

$$
m^{\text {ren }}=\frac{m-\Sigma(0,0,0)}{\left(Z_{1} Z_{2} Z_{3}\right)^{\frac{1}{3}}}
$$

In the final step, we will study the dynamics of constant couplings $\lambda_{\epsilon}$, governed by the $\beta_{\epsilon}$-functions ciphered by the following equations

$$
\lambda_{\epsilon}^{\mathrm{ren}}=-\frac{\Gamma_{4, \epsilon}(0,0,0,0,0,0)}{\left(Z_{1} Z_{2} Z_{3}\right)^{\frac{2}{3}}}, \quad \epsilon=1,2,3 .
$$

where $\Gamma_{4, \epsilon}\left(b_{1}, b_{2}, b_{3}, b_{1}^{\prime}, b_{2}^{\prime}, b_{3}^{\prime}\right)$ is the amputated 1PI four-point function.

Let us recall that the amputated 1PI four-point functions occurring in 69 read

$$
\begin{aligned}
& \Gamma_{4,1}\left(b_{1}, b_{2}, b_{3}, b_{1}^{\prime}, b_{2}^{\prime}, b_{3}^{\prime}\right)=\left\langle\varphi_{b_{1} b_{2} b_{3}} \bar{\varphi}_{b_{1}^{\prime} b_{2} b_{3}} \varphi_{b_{1}^{\prime} b_{2}^{\prime} b_{3}^{\prime}} \bar{\varphi}_{b_{1} b_{2}^{\prime} b_{3}^{\prime}}\right\rangle_{1 P I}^{t}, \\
& \Gamma_{4,2}\left(b_{1}, b_{2}, b_{3}, b_{1}^{\prime}, b_{2}^{\prime}, b_{3}^{\prime}\right)=\left\langle\varphi_{b_{1} b_{2} b_{3}} \bar{\varphi}_{b_{1} b_{2}^{\prime} b_{3}} \varphi_{b_{1}^{\prime} b_{2}^{\prime} b_{3}^{\prime}} \bar{\varphi}_{b_{1}^{\prime} b_{2} b_{3}^{\prime}}\right\rangle_{1 P I}^{t}, \\
& \Gamma_{4,3}\left(b_{1}, b_{2}, b_{3}, b_{1}^{\prime}, b_{2}^{\prime}, b_{3}^{\prime}\right)=\left\langle\varphi_{b_{1} b_{2} b_{3}} \bar{\varphi}_{b_{1} b_{2} b_{3}^{\prime}} \varphi_{b_{1}^{\prime} b_{2}^{\prime} b_{3}^{\prime}} \bar{\varphi}_{b_{1}^{\prime} b_{2}^{\prime} b_{3}}\right\rangle_{1 P I}^{t},
\end{aligned}
$$

where external indices, even though repeated, are not summed and follow the pattern of the vertices of the model. This is justified by the renormalization prescription.

Considering the reduced cases (ii) and (iii), there are potentially two wave function renormalizations $Z_{\epsilon=1,2}$. Nevertheless, the cancellation of one or many couplings may have drastic consequences on the way that the $\beta$-function equations have to be written. We will 
deal with these after computing the self-energy as well as the $\Gamma^{4}$-function in full generality and then putting to zero some of the contributions.

For $\epsilon, \check{\epsilon}, \check{\breve{\epsilon}}=1,2,3$, and pairwise distinct, we introduce the following formal sums:

$$
\begin{aligned}
\mathcal{S}_{\epsilon}(b) & :=\sum_{p_{1}, p_{2} \in \mathbb{Z}} 1 /\left(a_{\epsilon}|b|+a_{\check{\epsilon}}\left|p_{1}\right|+a_{\check{\epsilon}}\left|p_{2}\right|+m\right) \\
\mathcal{S}_{\epsilon}^{\prime}\left(b, b^{\prime}\right) & :=\sum_{p \in \mathbb{Z}} 1 /\left(a_{\check{\epsilon}}|b|+a_{\check{\epsilon}}\left|b^{\prime}\right|+a_{\epsilon}|p|+m\right) \\
S_{\epsilon} & :=\sum_{p_{1}, p_{2} \in \mathbb{Z}} 1 /\left(a_{\check{\epsilon}}\left|p_{1}\right|+a_{\check{\epsilon}}\left|p_{2}\right|+m\right)^{2}, \\
S_{1}^{\prime} & =\sum_{p_{1}, p_{2}} 1 /\left(a_{2}\left(\left|p_{1}\right|+\left|p_{2}\right|\right)+m\right)^{2}, \quad S_{2}^{\prime}=\sum_{p_{1}, p_{2}} 1 /\left(a_{1}\left|p_{1}\right|+a_{2}\left|p_{2}\right|+m\right)^{2}, \\
\mathcal{S}_{\epsilon}\left(b, b^{\prime}\right) & :=\sum_{p_{1}, p_{2}} 1 /\left[\left(a_{\epsilon}|b|+a_{\check{\epsilon}}\left|p_{1}\right|+a_{\check{\epsilon}}\left|p_{2}\right|+m\right)\left(a_{\epsilon}\left|b^{\prime}\right|+a_{\check{\epsilon}}\left|p_{1}\right|+a_{\check{\epsilon}}\left|p_{2}\right|+m\right)\right]
\end{aligned}
$$

Note that $\mathcal{S}_{\epsilon}$ is linearly divergent whereas $\mathcal{S}_{\epsilon}^{\prime}, S_{\epsilon}, S_{1,2}^{\prime}$ and $\underline{\mathcal{S}}_{\epsilon}$ are logarithmically divergent. Another important fact to notice is $\underline{\mathcal{S}}_{\epsilon}(0,0)=S_{\epsilon}$.

Let us prove the following proposition

Lemma 5. At one-loop, the self-energy and wave function renormalizations are given by

$$
\begin{aligned}
\Sigma\left(b_{1}, b_{2}, b_{3}\right) & =-\sum_{\epsilon} \lambda_{\epsilon} \mathcal{S}_{\epsilon}\left(b_{\epsilon}\right)-\lambda_{1} \mathcal{S}_{1}^{\prime}\left(b_{2}, b_{3}\right)-\lambda_{2} \mathcal{S}_{2}^{\prime}\left(b_{1}, b_{3}\right)-\lambda_{3} \mathcal{S}_{3}^{\prime}\left(b_{1}, b_{2}\right)+O\left(\lambda^{2}\right), \\
Z_{\epsilon} & =1-\lambda_{\epsilon} S_{\epsilon}+O\left(\lambda^{2}\right), \quad \epsilon=1,2,3
\end{aligned}
$$

where $O\left(\lambda^{2}\right)$ is a O-function of any quadratic products of any coupling constants $\lambda_{1,2,3}$.

Proof. We can first evaluate the self-energy (67) as

$$
\Sigma\left(b_{1}, b_{2}, b_{3}\right)=\sum_{\mathcal{G}} K_{\mathcal{G}} A_{\mathcal{G}}\left(b_{1}, b_{2}, b_{3}\right)
$$

where $\mathcal{G}$ runs over two-point 1 PI graphs with amplitude $A_{\mathcal{G}}\left(b_{1}, b_{2}, b_{3}\right)$ and corresponding combinatorial weight $K_{\mathcal{G}}$. The latter is known to be the number of Wick contractions given rise to $\mathcal{G}$. At one-loop, only the tadpole graphs $T_{1}, T_{2}, T_{3}$ (such that $\left.\sum_{J} g_{\widetilde{J}}=0\right) T_{1}^{\prime}, T_{2}^{\prime}$ and $T_{3}^{\prime}$ (such that $\sum_{J} g_{\widetilde{J}}=1$ ) (Fig.9 provides $T_{1}$ and $T_{1}^{\prime}$, the remaining configurations can be easily recovered by color permutations corresponding to the other configurations of the vertex $\left.\varphi^{4}\right)$ contribute to 77 with the combinatorial factors

$$
K_{T_{\epsilon}}=2=K_{T_{\epsilon}^{\prime}}, \quad \epsilon=1,2,3 .
$$

One gets the amplitude for each tadpole $T_{\epsilon}$

$$
A_{T_{\epsilon}}\left(b_{\epsilon}\right)=-\frac{\lambda_{\epsilon}}{2} \mathcal{S}_{\epsilon}\left(b_{\epsilon}\right)
$$

where $\mathcal{S}_{\epsilon}\left(b_{\epsilon}\right)$ is given by (71). Meanwhile, for $T_{\epsilon}^{\prime}$, we have the amplitude

$$
A_{T_{\epsilon}^{\prime}}\left(b_{\check{\epsilon}}, b_{\check{\epsilon}}\right)=-\frac{\lambda_{1}}{2} \mathcal{S}_{\epsilon}^{\prime}\left(b_{\check{\epsilon}}, b_{\check{\epsilon}}\right)
$$



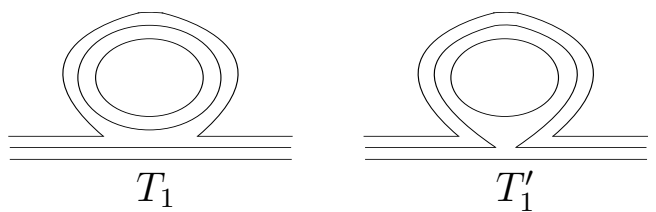

Figure 9: Tadpoles for the first type of vertex.

with $\mathcal{S}_{\epsilon}^{\prime}\left(b_{\check{\epsilon}}, b_{\check{\epsilon}}\right)$ given as $(72)$. At first order, we obtain the self-energy as the sum of these contributions as

$$
\Sigma\left(b_{1}, b_{2}, b_{3}\right)=-\sum_{\epsilon} \lambda_{\epsilon} \mathcal{S}_{\epsilon}\left(b_{\epsilon}\right)-\lambda_{1} \mathcal{S}_{1}^{\prime}\left(b_{2}, b_{3}\right)-\lambda_{2} \mathcal{S}_{2}^{\prime}\left(b_{1}, b_{3}\right)-\lambda_{3} \mathcal{S}_{3}^{\prime}\left(b_{1}, b_{2}\right)+O\left(\lambda^{2}\right) .
$$

It should be emphasized that not all contributions of $\Sigma\left(b_{1}, b_{2}, b_{3}\right)$ have to be taken into account for the wave function renormalizations (66). Only those leading to a log-divergent behavior after differentiation have to be considered. A quick inspection shows that $\mathcal{S}_{\epsilon}^{\prime}\left(b, b^{\prime}\right)$ are log-divergent hence should be only considered for the mass renormalization. The wave function renormalizations (66) can be finally expressed as

$$
Z_{\epsilon}=1-\lambda_{\epsilon} S_{\epsilon}+O\left(\lambda^{2}\right)
$$

where $S_{\epsilon}$ is given by 73$)$.

We are in position to compute the dynamics of the renormalized wave coupling constants $a_{\epsilon}^{\text {ren }}$ and of the renormalized mass $m^{\text {ren }}$. In the same previous notations, the following statement holds:

Theorem 4. At first order, the renormalized wave function couplings and mass satisfy, respectively,

$$
\begin{aligned}
& a_{\epsilon}^{r e n}=a_{\epsilon}\left[1-\frac{1}{3}\left(2 \lambda_{\epsilon} S_{\epsilon}-\lambda_{\check{\epsilon}} S_{\check{\epsilon}}-\lambda_{\check{\epsilon}} S_{\check{\epsilon}}\right)\right]+O\left(\lambda^{2}\right), \quad \epsilon=1,2,3, \check{\epsilon} \neq \epsilon, \check{\epsilon} \neq \check{\check{\epsilon}} \neq \epsilon,(83) \\
& m^{r e n}=m+\sum_{\epsilon=1}^{3} \lambda_{\epsilon} \mathcal{S}_{\epsilon}(0)+O\left(\lambda^{2}, \lambda \ln \Lambda\right) .
\end{aligned}
$$

for momentum cut-off $\Lambda$.

Proof. Concerning the first statement (83), by simple index permutations, all cases can be easily deduced from $\epsilon=1$ hence we will focus only on this situation. Using Lemma 5 and remaining at first order in the constant couplings $\lambda_{\epsilon}$, we have:

$$
a_{1}^{\text {ren }}=a_{1}\left(\frac{1-2 \lambda_{1} S_{1}+O\left(\lambda^{2}\right)}{1-\left(\lambda_{2} S_{2}+\lambda_{3} S_{3}\right)+O\left(\lambda^{2}\right)}\right)^{\frac{1}{3}}=a_{1}\left[1-\frac{1}{3}\left(2 \lambda_{1} S_{1}-\lambda_{2} S_{2}-\lambda_{3} S_{3}\right)\right]+O\left(\lambda^{2}\right) .
$$

Focusing on the renormalized mass equation (68), Lemma 5 allows us to write

$$
m^{\mathrm{ren}}=\frac{m+\sum_{\epsilon} \lambda_{\epsilon} \mathcal{S}_{\epsilon}(0)+\lambda_{1} \mathcal{S}_{1}^{\prime}(0,0)+\lambda_{2} \mathcal{S}_{2}^{\prime}(0,0)+\lambda_{3} \mathcal{S}_{3}^{\prime}(0,0)+O\left(\lambda^{2}\right)}{1-\frac{1}{3}\left(\lambda_{1} S_{1}+\lambda_{2} S_{2}+\lambda_{3} S_{3}\right)+O\left(\lambda^{2}\right)}
$$




$$
\begin{aligned}
= & m+\sum_{\epsilon} \lambda_{\epsilon} \mathcal{S}_{\epsilon}(0)+\lambda_{1} \mathcal{S}_{1}^{\prime}(0,0)+\lambda_{2} \mathcal{S}_{2}^{\prime}(0,0)+\lambda_{3} \mathcal{S}_{3}^{\prime}(0,0) \\
& +\frac{1}{3}\left(\lambda_{1} S_{1}+\lambda_{2} S_{2}+\lambda_{3} S_{3}\right)+O\left(\lambda^{2}\right) .
\end{aligned}
$$

Neglecting the subleading divergences compared to the linear divergence of $\mathcal{S}_{\epsilon}(0)$, one is led to (84) after having introduced a cut-off $\Lambda$ in the sums $\mathcal{S}_{\epsilon}^{\prime}(0,0)$ and $S_{\epsilon}$.

Discussion. The $\gamma$-functions of the model can be defined by restricting the space of the couplings parameters to a smaller subspace. In fact, the reduction will be performed on the space of six couplings $\lambda_{1,2,3}$ and $a_{1,2,3}$.

Many cases may occur by collapsing couplings. The first case is straightforward

(i) We set $a_{\epsilon}=a$ in 83 . This enforces $\lambda_{1}=\lambda_{2}=\lambda_{3}$ and induces a unique equation:

$$
a^{\text {ren }}=a .
$$

The latter means that the wave function coupling becomes stationary in the UV. In other words, the $\gamma$-function for this case is trivial:

$$
\gamma=0
$$

Meanwhile, from (84), one infers

$$
\beta_{m}=-3
$$

(ii) Otherwise, setting $\lambda_{2}=\lambda_{3}=0$ but $\lambda_{1} \neq 0$ yields necessarily that $a_{1} \neq a_{2}$. We need to re-evaluate the self-energy from (81) and we obtain the only non trivial wave function renormalization

$$
Z_{1}^{\left({ }^{\prime}\right)}=1-\lambda_{1} S_{1}^{\prime}+O\left(\lambda_{1}^{2}\right)
$$

where $S_{1}^{\prime}$ is given by $(74)$. The equations of the couplings find some modified form:

$$
a_{1}^{\left({ }^{\prime}\right) \text { ren }}=a_{1}, \quad a_{2}^{\left({ }^{\prime}\right) \text { ren }}=a_{2} \frac{1}{Z_{1}}=a_{2}\left(1+\lambda_{1} S_{1}^{\prime}\right)+O\left(\lambda_{1}^{2}\right) .
$$

The $\gamma$-functions at one-loop in this restricted space are given by

$$
\gamma_{1}^{\left({ }^{\prime}\right)}=0, \quad \gamma_{2}^{\left({ }^{\prime}\right)}=-1
$$

Assuming that the sign of $\lambda_{1}$ is positive, $a_{1}$ has a stable value in the UV, whereas $a_{2}$ flows towards a vanishing value. Under the same assumptions, we get for the mass, the renormalized mass equation and $\beta_{m}$ function

$$
m^{\left({ }^{\prime}\right) \text { ren }}=m+\lambda_{1} \tilde{\mathcal{S}}_{1}(0)+O\left(\lambda_{1}^{2}, \lambda_{1} \ln \Lambda\right), \quad \beta_{m}^{\left({ }^{\prime}\right)}=-1
$$

where $\tilde{\mathcal{S}}_{1}(0)$ can be inferred from $\mathcal{S}_{1}(0)$ after identifying $a_{2}=a_{3}$.

(iii) Setting $\lambda_{2}=\lambda_{3} \neq 0, \lambda_{1}=0$ and $a_{1} \neq a_{2}$ and computing the self-energy, one has the unique non trivial wave function renormalization

$$
Z_{2}^{(\prime \prime)}=1-\lambda_{2} S_{2}^{\prime}+O\left(\lambda_{2}^{2}\right)
$$


where $S_{2}^{\prime}$ is provided by $(74)$. In this restricted space, the coupling equations are given by

$$
a_{2}^{\left({ }^{\prime \prime}\right) \text { ren }}=a_{2}, \quad a_{1}^{\left({ }^{\prime \prime}\right) \text { ren }}=a_{1} \frac{1}{Z_{2}^{\left({ }^{\prime \prime}\right)}}=a_{1}\left(1+\lambda_{2} S_{2}^{\prime}\right)+O\left(\lambda_{2}^{2}\right),
$$

yielding the $\gamma$-functions at one-loop

$$
\gamma_{2}^{(\prime \prime)}=0, \quad \gamma_{1}^{(\prime \prime)}=-1
$$

The same assumptions give for the mass

$$
\beta_{m}^{\left({ }^{\prime \prime}\right)}=-1
$$

Thus cases (ii) and (iii) share similar properties.

Theorem 5. The renormalizable coupling constants associated with $\lambda_{\epsilon}$, for $\epsilon=1,2,3$, satisfy

$$
\lambda_{\epsilon}^{r e n}=\lambda_{\epsilon}+\lambda_{\epsilon}\left[-\lambda_{\epsilon} S_{\epsilon}+\frac{2}{3}\left(\lambda_{1} S_{1}+\lambda_{2} S_{2}+\lambda_{3} S_{3}\right)\right]+O\left(\lambda^{3}\right),
$$

where $O\left(\lambda^{3}\right)$ is a O-function of any cubic product of all couplings $\lambda_{1,2,3}$.

Proof. The one-loop 1PI four-point functions can be written as

$$
\Gamma_{4, \epsilon}\left(b_{1}, b_{2}, b_{3}, b_{1}^{\prime}, b_{2}^{\prime}, b_{3}^{\prime}\right)=\sum_{\mathcal{G}} K_{\mathcal{G}} \mathcal{A}_{\mathcal{G}}\left(b_{1}, b_{2}, b_{3}, b_{1}^{\prime}, b_{2}^{\prime}, b_{3}^{\prime}\right)
$$

where $\mathcal{G}$ is a four-point 1PI graph with the topology as required by the renormalization, with amplitude $\mathcal{A}_{\mathcal{G}}\left(b_{1}, b_{2}, b_{3}, b_{1}^{\prime}, b_{2}^{\prime}, b_{3}^{\prime}\right)$ and combinatorial weight $K_{\mathcal{G}}$.

Note that, interestingly, the "mixed" graphs obtained by gluing vertices of different kind do not contribute to the effective coupling constants. Indeed, although these graphs could form melons, they all possess two lines and one face giving a convergent power-counting $M^{-1}$. Another way to agree with this fact is to observe that the boundary graph (even though melonic) of any of them is disconnected with $C_{\partial \mathcal{G}}=2$. As a consequence, in the following developments, cross terms involving product of coupling constants $\lambda_{\epsilon} \lambda_{\epsilon^{\prime}}, \epsilon \neq \epsilon^{\prime}$, are inexistent. Hence, a unique graph $F_{\epsilon}$ contribute to $\Gamma_{4, \epsilon}$ (Fig.10 displays such a contribution for $\epsilon=1$, the remaining can be deduced by permutations) and its combinatorial factor is always

$$
K_{F_{\epsilon}}=2 \cdot 2 \cdot 2 \text {. }
$$

Given an $\epsilon$, the amplitude of each graph is such that

$$
\mathcal{A}_{F_{\epsilon}}\left(b_{\epsilon}, b_{\epsilon}^{\prime}\right)=\frac{\lambda_{\epsilon}^{2}}{2^{2} 2 !} \underline{\mathcal{S}}_{\epsilon}\left(b_{\epsilon}, b_{\epsilon}^{\prime}\right),
$$

where $\underline{\mathcal{S}}_{\epsilon}\left(b_{\epsilon}, b_{\epsilon}^{\prime}\right)$ is given by 75 .

The amputated 1PI four-point functions $\Gamma_{4, \epsilon=1,2,3}$ defined by 70 and $(99)$ can be evaluated at one-loop as

$$
\Gamma_{4, \epsilon}\left(b_{1}, b_{2}, b_{3}, b_{1}^{\prime}, b_{2}^{\prime}, b_{3}^{\prime}\right)=-\lambda_{\epsilon}+\lambda_{\epsilon}^{2} \underline{\mathcal{S}}_{\epsilon}\left(b_{\epsilon}, b_{\epsilon}^{\prime}\right)+O\left(\lambda^{3}\right)
$$




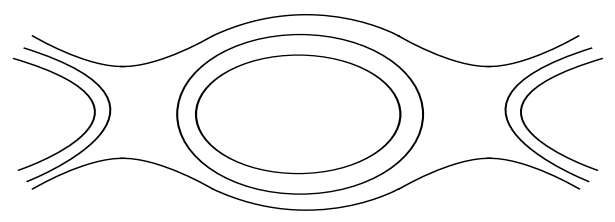

Figure 10: The unique melonic 1-loop four-point function with melonic connected boundary for the first type of vertex.

where $O\left(\lambda^{3}\right)$ is a O-function of any cubic product of coupling constants. Therefore, at low external momenta, they reduce to

$$
\Gamma_{4, \epsilon}(0,0,0,0,0,0)=-\lambda_{\epsilon}+\lambda_{\epsilon}^{2} S_{\epsilon}+O\left(\lambda^{3}\right) .
$$

Having all required ingredients, we are in position to evaluate the ratios (69). Using Lemma 5 and (103), by direct algebra, one comes to

$$
\begin{aligned}
\lambda_{\epsilon}^{\mathrm{ren}} & =-\frac{-\lambda_{\epsilon}+\lambda_{\epsilon}^{2} S_{\epsilon}+O\left(\lambda^{3}\right)}{\left\{\prod_{\epsilon}\left(1-\lambda_{\epsilon} S_{\epsilon}+O\left(\lambda^{2}\right)\right)\right\}^{\frac{2}{3}}} \\
& =\lambda_{\epsilon}+\lambda_{\epsilon}\left(-\lambda_{\epsilon} S_{\epsilon}+\frac{2}{3}\left(\lambda_{1} S_{1}+\lambda_{2} S_{2}+\lambda_{3} S_{3}\right)\right)+O\left(\lambda^{3}\right)
\end{aligned}
$$

which achieves the proof.

Discussion. We can once again discuss the merging of coupling constants $\lambda_{1,2,3}$ into specific varieties in order to deduce the $\beta$-functions.

(i) We merge all couplings such that $a_{\epsilon}=a$ and $\lambda_{\epsilon}=\lambda$, such that $S_{\epsilon}(73)$ becomes

$$
\left.S=\sum_{p_{1}, p_{2} \in \mathbb{Z}} 1 /\left[a\left(\left|p_{1}\right|+\left|p_{2}\right|\right)+m\right)\right]^{2}
$$

then we have from 98

$$
\lambda^{\text {ren }}=\lambda+\lambda^{2} S+O\left(\lambda^{3}\right) .
$$

such that the $\beta$-function of the model with single wave function renormalization and single coupling constant is given by

$$
\beta=-1
$$

This model is therefore asymptotically free.

(ii) Assuming that $a_{1} \neq a_{2}=a_{3}$, and $\lambda_{1} \neq 0=\lambda_{2,3}$, there is a unique equation for the coupling constant $\lambda_{1}$ expressed as

$$
\lambda_{1}^{\left({ }^{\prime}\right) \text { ren }}=-\frac{\Gamma_{4,1}^{\left({ }^{\prime}\right)}}{\left(Z_{1}^{\left({ }^{\prime}\right)}\right)^{2}}=-\frac{\left.\left(-\lambda_{1}+\lambda_{1}^{2} S_{1}^{\prime}+O\left(\lambda_{1}^{3}\right)\right)\right)}{\left(1-\lambda_{1} S_{1}^{\prime}+O\left(\lambda_{1}^{2}\right)\right)^{2}}=\lambda_{1}+\lambda_{1}^{2} S_{1}^{\prime}+O\left(\lambda_{1}^{3}\right) .
$$

From this, we infer

$$
\beta_{1}^{(\prime)}=-1
$$

Consequently, the model is also asymptotically free. 
(iii) Last, we set $\lambda_{2}=\lambda_{3} \neq 0, \lambda_{1}=0$ and $a_{1} \neq a_{2}$. The coupling constant equation can be written

$$
\lambda_{2}^{\left({ }^{\prime \prime}\right) \text { ren }}=-\frac{\Gamma_{4,2}^{\left({ }^{\prime \prime}\right)}}{\left(Z_{2}^{(\prime \prime}\right)^{2}}
$$

and yields in the same way as previously done in (108) the one-loop $\beta$-function

$$
\beta_{2}^{(\prime)}=-1
$$

showing that this theory is asymptotically free.

\subsection{RG flows}

We restrict the RG flow equations to the particular situations discussed so far. It turns out that for these cases, explicit solutions are affordable at this truncation. Cases (i) and (ii) will be only discussed for simplicity.

Case (i): $\lambda_{\epsilon}=\lambda$ and $a_{\epsilon}=a$. This case yields the coupling equations (87) and (106) of the form:

$$
a_{\epsilon}^{\text {ren }}=a, \quad \lambda^{\text {ren }}=\lambda+\lambda^{2} S+O\left(\lambda^{3}\right) .
$$

Hence, the wave function renormalization is stable whereas the coupling constant satisfies the discrete RG equation (truncated at first order)

$$
\lambda_{i-1}=\lambda_{i}+\lambda_{i}^{2} S_{i}
$$

where $S_{i}$ is given from $S(105)$ by restricting the sum to high momenta $\left|p_{1}\right|+\left|p_{2}\right| \in\left[M^{i-1}, M^{i}\right]$ such that (see Appendix $B$ for more details)

$$
S_{i}=\frac{4}{a_{i}^{2}} \kappa_{M}+O\left(M^{-i}\right),
$$

with $\kappa_{M}$ a log-divergent term in $M$ which does not depend on the scale $i$. We get the solution, using $a_{i}=a$,

$$
\frac{d \lambda_{i}}{\lambda_{i}^{2}}=-\frac{4}{a^{2}} \kappa_{M} d i, \quad \lambda(i)=\frac{a^{2} \lambda_{u v}}{a^{2}-4 \kappa_{M}(\Lambda-i) \lambda_{u v}}
$$

where we have introduced the coupling value $\lambda_{u v}$ at the cut-off scale $\Lambda$. The RG flow of $\lambda$ is pictured in Fig.11.

Case (ii): $\lambda_{2}=\lambda_{3}=0 \neq \lambda_{1}$ and $a_{2}=a_{3} \neq a_{1}$. The RG flow equations can be obtained from (91) and (108) as

$$
\begin{aligned}
& a_{1}^{\text {ren }}=a_{1}, \quad a_{2}^{\text {ren }}=a_{2}\left(1+\lambda_{1} S_{1}^{\prime}\right)+O\left(\lambda_{1}^{2}\right), \\
& \lambda_{1}^{\text {ren }}=\lambda_{1}+\lambda_{1}^{2} S_{1}^{\prime}+O\left(\lambda_{1}^{3}\right),
\end{aligned}
$$

with $S_{1}^{\prime}$ given by 74 . The same RG flow equations (truncated at one loop corrections) translate in discrete version at scale $i$ as

$$
a_{1 ; i-1}=a_{1 ; i}, \quad a_{2 ; i-1}=a_{2 ; i}\left[1+\lambda_{1 ; i} S_{1, i}^{\prime}\right], \quad \lambda_{1 ; i-1}=\lambda_{1 ; i}+\lambda_{1 ; i}^{2} S_{1 ; i}^{\prime} .
$$




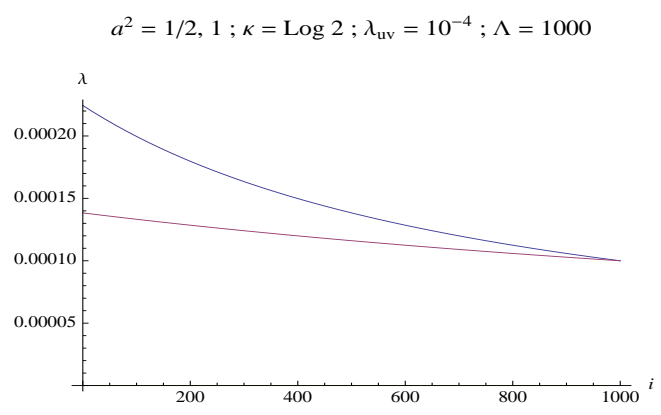

Figure 11: $\quad$ RG flow of the coupling constant $\lambda$ running to a trivial fixed point for two values $a=1 / 2$ (top) and $a=1$ (bottom) and for a UV cut-off $\Lambda=1000$ and other parameters fixed such that $\lambda_{u v}=10^{-4}$ and $\kappa=\log M=\log 2$.

Summing $S_{1}^{\prime}$ only on high momenta $\left|p_{1}\right|+\left|p_{2}\right| \in\left[M^{i-1}, M^{i}\right]$, we have $S_{1 ; i}^{\prime}=4 / a_{2 ; i}^{2} \kappa_{M}+$ $O\left(M^{-i}\right)$. Focusing of the second and third expressions, these discrete equations can be put, at first order, in an equivalent differential form

$$
\begin{aligned}
\frac{d a_{2 ; i}}{d i} & =-4 \frac{1}{a_{2 ; i}} \lambda_{1 ; i} \kappa_{M} \\
\frac{d \lambda_{1 ; i}}{d i} & =-4 \frac{1}{a_{2 ; i}^{2}} \lambda_{1 ; i}^{2} \kappa_{M}
\end{aligned}
$$

which induces that

$$
\lambda_{1 ; i} / a_{2 ; i}=\tilde{K}
$$

for some constant $\tilde{K}$. We substitute $\tilde{K}$ in $(118)$ and get

$$
d \lambda_{1 ; i}=-4 \kappa_{M} \tilde{K}^{2} d i \quad \Leftrightarrow \quad \lambda_{1}(i)=\lambda_{u v}+4 \kappa_{M} \tilde{K}^{2}(\Lambda-i) .
$$

Thus, one can readily obtain $a_{2}(i)$ by combining $(120)$ and $(119)$. We see that the flow of this model actually differs from the previous one. Both couplings $\lambda_{1}$ and $a_{2}$ go linearly to their UV fixed values.

\section{Conclusion}

We have shown that a rank 3 tensor model on $U(1)^{3}$, analogue of the previous model identified in [45], is perturbatively renormalizable at all orders of perturbation theory. The proof of this statement relies on both combinatorics and the colored model properties serving as underpinning of the present class of tensor models. The interplay of three ingredients, namely a slice decomposition, a power-counting theorem and a generalized locality principle has guided us towards a proof of renormalizability of this model. We have also introduced different wave function couplings for each strand hence giving them a different dynamics as well as different interaction coupling constants. This definitely enlarges the class of renormalizable models of the kind. As it should be also emphasized, the renormalization has been performed in the momentum space and not in the direct space. This particular basis has allowed us 
to identify an analogue theorem for the momentum routine useful for renormalization in ordinary quantum field theory [52]. The second part of this contribution was devoted to the computations of the $\gamma$-, $\beta$-functions and the corresponding RG flow of couplings constants in the theory. There exist two classes of underlying models turn out to be asymptotically free in the UV limit: (a) The model obtained by merging all coupling constants, such that $\lambda_{1,2,3}=\lambda$ and $a_{1,2,3}=a$; (b) the class of models defined by $\lambda_{2,3}=0 \neq \lambda_{1}$ and $a_{1} \neq a_{2}=a_{3}$ and the symmetric obtained by color permutation.

The model introduced here and its analogue developed in 45 claimed to be simplified but possible models for quantum gravity using the "tensor device" [46]. What we have shown here, by exhibiting such a renormalizable and asymptotically free (in the UV) model, is that the tensor approach for quantum gravity is a promising line of research for those who believe that gravity should be described by a renormalizable quantum field theory.

Future prospects are can be performed in different directions. Nonlocal tensor models over copies of $\mathbb{R}$ can be treated provided one introduces an IR regulator. For instance, the Mehler propagator $1 /\left(p^{2}+x^{2}\right)$ (on each strand) could be an interesting proposal. The resulting models should be called tensor extension of the Grosse-Wulkenhaar model [48] in noncommutative field theory. Another important question is whether or not such an analysis could be handled for GFT models on copies of $S U(2)$. A priori, since the tensor $1 / N$ expansion is also valid for GFT models, the answer is yes. Nevertheless, the level of difficulty is much higher is that situation. The renormalization will definitely involve both $S U(2)$ recoupling theory and Taylor expansions of graphs around their local parts. These later entail generalized saddle point analysis which are far to be easy for general graphs at any order of perturbation theory [31. Besides, going deeper in the analysis of the class of models presented here, an important point would be to investigate if the rank four model shares this important property of asymptotic freedom. Finally, interesting though more abstract questions can be also addressed at this stage. For instance, the determination of topological polynomials associated with the graphs of these models using an extended (i.e. tensor) parametric representation of graph amplitudes in the spirit of [59] could be a fruitful line of investigation.

\section{Appendix}

\section{A Proof of Lemma 3 and divergent graph classification}

\section{A.1 Proof of Lemma 3}

This appendix provides the proof of Lemma 3 using a sequence of $0 k$-dipole contractions. First of all, we need to define a central notion which is the following:

Definition 3 (Pairs). Let $\mathcal{G}_{\text {color }}$ be a colored graph and consider a $0 k$-dipole inside $\mathcal{G}_{\text {color }}$. A "pair" is a couple of colors $(a b), a, b=0,1,2,3$. A pair is called "outer" if the two colors are external to the dipole. A pair which has one color inside the dipole and one out is called a "mixed" pair. A pair with two colors inside the dipole is called an "inner" pair. A pair (ab) is said to belong to a jacket if the pair is one of the four adjacent (consecutive) pairs in the jacket cycle (0xyz). 

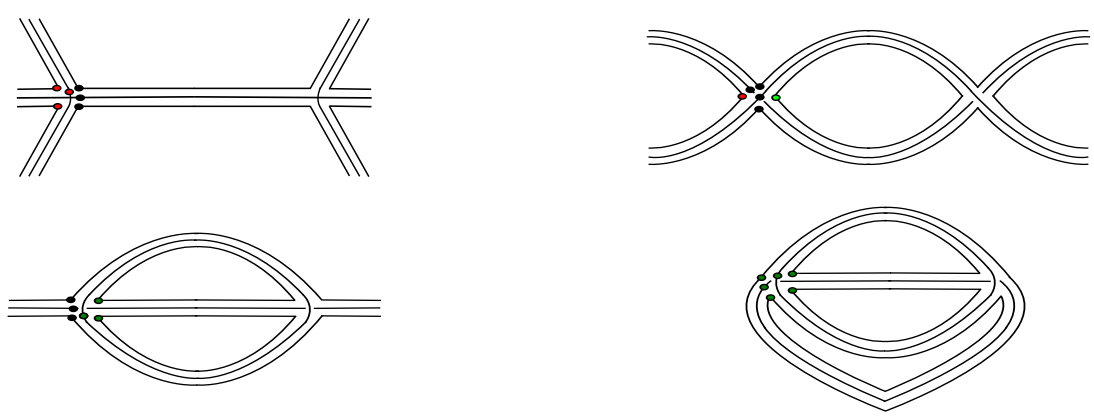

Figure 12: Different types of pairs highlighted for each $0 k$-dipoles: External pairs (in red), internal pairs (in green) and mixed pairs (in black).

The total number of pairs is always 6 and the number of mixed pairs is at least 3. Fig.12 indicates the different kinds of pairs.

Contracting a dipole leads to different cases and we urge to further classify the different pairs in a more concise manner. An outer pair is said of type A, or disconnected by the dipole contraction if the half-strands at each corner on the left and on the right of the dipole belong to two different connected components of the graph after the contraction. Otherwise, we call it a "special" pair. A special pair can be single-faced if the two corners belong to the same face of the graph, or double-faced if the two corners belong to two different faces of the graph. Any type A outer pair should be single-faced at the beginning. We therefore classify outer pairs in the following three types:

- Type A outer pairs are single-faced,

- Type B outer pairs are single-faced,

- Type C outer pairs are double-faced.

Transverse pairs are mixed pairs which do not change their number of faces under contraction. Inner pairs have one face less after contraction. Type A and B outer pairs have one face more after contraction whereas type $\mathrm{C}$ external pairs have one face less after the contraction. In summary, for any jacket $\widetilde{J}$, we can relate the number of faces before and after contraction by the formula

$$
F_{\widetilde{J}}-F_{\widetilde{J}}=\left|A_{\widetilde{J}}\right|+\left|B_{\widetilde{J}}\right|-\left|C_{\widetilde{J}}\right|-\left|I_{\widetilde{J}}\right|
$$

where $\left|X_{\widetilde{J}}\right|$ is the number of pairs of type $\mathrm{X}=\mathrm{A}, \mathrm{B}, \mathrm{C}$, in the jacket, and $\left|I_{\widetilde{J}}\right|$ is the number of inner faces.

Lemma 3 states that, for a single jacket $\widetilde{J}$, we have

$$
g_{\widetilde{J}}-g_{\widetilde{J}^{\prime}} \geq 0
$$

We emphasize that the proof of this statement which will be given here slightly differs from the proof of a related statement as found in [45]. Indeed, in the latter reference, the sum over jackets is performed at the level of A.2 and leads directly to a bound on $\sum_{j}\left(g_{\widetilde{J}}-g_{\widetilde{J}^{\prime}}\right)$. 


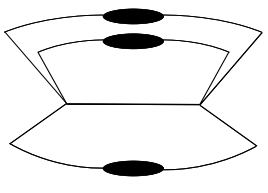

A

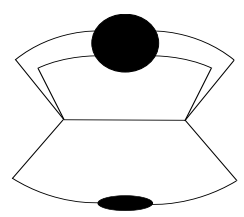

B

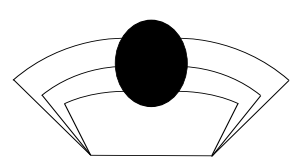

C

Figure 13: 00-dipoles configurations.

However, as was mentioned [45] and was computed in a earlier version of the same paper, the relation $\mathrm{A} .2$ is a stronger statement than the result on the sum of jackets and therefore worth to be communicated.

Proof of Lemma 3. We first translate the difference between genera of jackets before and after contraction in terms of the basic combinatorial elements:

$$
\begin{aligned}
2-2 g_{\widetilde{J}} & =V-L+F_{\widetilde{J}}, \\
2 c^{\prime}-2 g_{\widetilde{J}^{\prime}} & =V^{\prime}-L^{\prime}+F_{\widetilde{J}^{\prime}}=(V-2)-(L-4)+F_{\widetilde{J}^{\prime}}, \\
\left(g_{\widetilde{J}}-g_{\widetilde{J}^{\prime}}\right) & =\frac{1}{2}\left[\left(F_{\widetilde{J}^{\prime}}-F_{\widetilde{J}}\right)+2-2\left(c^{\prime}-1\right)\right] .
\end{aligned}
$$

The sign of $g_{\widetilde{J}}-g_{\widetilde{J}^{\prime}}$ reduces to that of $\left(F_{\widetilde{J}^{\prime}}-F_{\widetilde{J}}\right)+2-2\left(c^{\prime}-1\right)$. The latter quantity proves to be always positive jacket by jacket. To prove this claim, we perform the following systematic analysis on the types of $0 k$-dipole contractions.

- 1rst Case: 00-dipole contraction. This case is defined by a contraction of a unique internal line with color 0 . In any initial colored graph, there are three mixed pairs, three outer pairs and no inner pair. Meanwhile, each jacket contains two mixed and two outer pairs.

- 1rst subcase $c^{\prime}=3$ (Fig. 13A). This situation happens if the initial graph has a connected two-point subgraph on each line 1,2,3. Consequently, all three outer pairs must be of type $A$. Hence, the 00-dipole contraction yields for all jackets:

$$
\left(F_{\widetilde{J}^{\prime}}-F_{\widetilde{J}}\right)+2-2\left(c^{\prime}-1\right)=0 .
$$

so that 46 is true.

- 2nd subcase $c^{\prime}=2$ (Fig.13B). This case happens if we have one connected two-point functions plus one connected four-point function on four half-lines hooked to the dipole. In that case, we have two corner pairs of type A and one special pair of type B or C. It can be seen that

$$
\left(F_{\widetilde{J}^{\prime}}-F_{\widetilde{J}}\right)+2-2\left(c^{\prime}-1\right)=2-2\left|C_{\widetilde{J}}\right| \cdot
$$

Hence in all cases, 46 is true.

- 3rd subcase $c^{\prime}=1$ (Fig.13C). Contracting the dipole gives $c^{\prime}-c=0$. This can happen when a connected six-point function is hooked to the dipole. There no type A corners and three special pairs. Each jackets contains at most two special pairs. Contracting the dipole yields

$$
\left(F_{\widetilde{J}^{\prime}}-F_{\widetilde{J}}\right)+2-2\left(c^{\prime}-1\right)=4-2\left|C_{\widetilde{J}}\right|,
$$

such that (46) is true. 


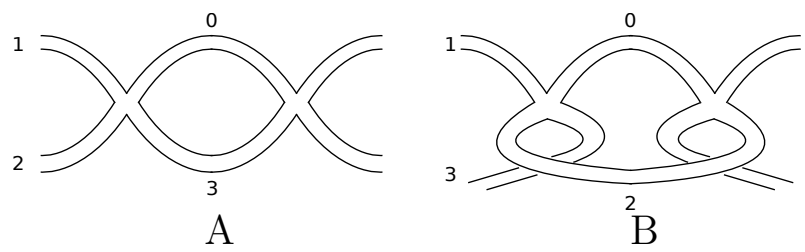

Figure 14: A jacket (0123) which contains the pair (03) defining the dipole (A) and another jacket (0123) from another dipole which does not contain the same pair.

- 2nd Case: 01-dipole contraction. There is one inner, four mixed and one outer pairs. A distinction should be made between inner-adjacent jackets, for which the inner pair of the dipole belongs to the jacket (see Fig $14 \mathrm{~A}$, for instance, the pair (03) belongs both to the jacket (0123) and the dipole) from non-inner-adjacent jackets for which it does not (see Fig 14 B, for instance, the pair (02) is not in the jacket (0123) but defines the dipole). We count two subcases:

- 1rst subcase $c^{\prime}=2$. In that case, the unique outer pair is of type A. An inner-adjacent jacket has two mixed pairs, one inner pair and one pair of type A. A non-inner-adjacent jacket has four mixed pairs, no inner pair and looses the type A pair. One finds, in all cases,

$$
\left(F_{\widetilde{J}^{\prime}}-F_{\widetilde{J}}\right)+2-2\left(c^{\prime}-1\right)=4-4=0,
$$

and $(46)$ is verified.

- 2nd subcase $c^{\prime}=1$. This case reduces to the presence of one special pair. An inner-adjacent jacket has two mixed pairs, one inner pair and one special. A non-inner-adjacent jacket has four mixed pairs, no inner pair and no special pair. In all cases,

$$
\left(F_{\widetilde{J}^{\prime}}-F_{\widetilde{J}}\right)+3-2\left(c^{\prime}-1\right)=2-2\left|C_{\widetilde{J}}\right|
$$

and 46 is true.

- 3rd Case: 02-dipole contraction. There are three inner pairs, three mixed pairs and no outer pair. Only the case $c^{\prime}=1$ occurs and one gets

$$
\left(F_{\widetilde{J}^{\prime}}-F_{\widetilde{J}}\right)+2-2\left(c^{\prime}-1\right)=0
$$

hence, 46) is satisfied.

- 4th Case: 03-dipole contraction. This case is straightforward and corresponds to the contraction of a vacuum connected component (with two vertices and four lines). There are six inner pairs, each jacket containing four of these pairs and we have $\left(c^{\prime}-1\right)=-1$. Thus

$$
\left(F_{\widetilde{J}^{\prime}}-F_{\widetilde{J}}\right)+2-2\left(c^{\prime}-1\right)=0 .
$$

Hence (46) is again valid. 


\section{A.2 Classification of divergent graphs}

We extract from the divergence degree data all graphs which could lead to $\omega_{d}(\mathcal{G}) \geq 0$ and aim at proving that Table 1 of Subsection 4.3 exhausts all possibilities.

Let us define the integer $P(\mathcal{G})=\left(C_{\partial \mathcal{G}}-1\right)+V_{2}+\frac{1}{2}\left[N_{\text {ext }}-4\right]$. We reformulate Lemma 4 in the following terms:

$$
\begin{aligned}
\omega_{d}(\mathcal{G})=-\left[\sum_{J} g_{\widetilde{J}}-g_{\partial \mathcal{G}}\right]-P(\mathcal{G}) & \leq-2 g_{\partial \mathcal{G}}-P(\mathcal{G}) \\
g_{\partial \mathcal{G}}=0 & \text { and } \sum_{J} g_{\widetilde{J}}>0 \quad \Rightarrow \quad \omega_{d}(\mathcal{G}) \leq-1-P(\mathcal{G}) .
\end{aligned}
$$

We now seek the list of graphs with $\omega_{d}(\mathcal{G}) \geq 0$ which are those which should be renormalized. Case $N_{\text {ext }}>4$ : Considering $N_{\text {ext }} \geq 6$ leads to $P(\mathcal{G}) \geq 1$. Thus, $\omega(\mathcal{G}) \leq-1$ and the graph amplitude is simply converging.

Case $N_{\text {ext }}=4$ : Under this condition, one has $P(\mathcal{G})=\left(C_{\partial \mathcal{G}}-1\right)+V_{2}$. The divergence degree is at most zero. The significant case occurs when $\omega(\mathcal{G})=0$ and for that one must have

$$
C_{\partial \mathcal{G}}=1, \quad g_{\partial \mathcal{G}}=\sum_{J_{\partial}} g_{J_{\partial}}=0, \quad V_{2}=0
$$

Case $N_{\text {ext }}=2$ : Since $C_{\partial \mathcal{G}} \leq N_{\text {ext }} / 2$, we should have $C_{\partial \mathcal{G}}=1$ and, therefore, $P(\mathcal{G})=V_{2}-1$. Furthermore, the only possible boundary colored graph made with two vertices is unique and does not have any genus $g_{\partial \mathcal{G}}=0$. We conclude that the divergence degree is at most 1 . One may have $\omega(\mathcal{G})=1$ if $P(\mathcal{G})=-1$, and only if

$$
C_{\partial \mathcal{G}}=1, \quad g_{\partial \mathcal{G}}=\sum_{J} g_{\widetilde{J}}=0, \quad V_{2}=0
$$

Besides, $\omega(\mathcal{G})=0$ can happen in two cases: $(1) P(\mathcal{G})=0$, in which case, we have

$$
C_{\partial \mathcal{G}}=1, \quad g_{\partial \mathcal{G}}=\sum_{J} g_{\widetilde{J}}=0, \quad V_{2}=1
$$

or $(2) P(\mathcal{G})=-1$ and, so,

$$
C_{\partial \mathcal{G}}=1, \quad g_{\partial \mathcal{G}}=0, \quad \sum_{J} g_{\widetilde{J}}=1, \quad V_{2}=0
$$

In summary, the divergent graphs are determined by the Table 1 of Subsection 4.3 .

\section{B Formal sum approximations}

We start by proving the claim (114) by giving the relevant part of the formal sum

$$
S=\sum_{p_{1}, p_{2} \in \mathbb{N}} \frac{1}{\left(p_{2}+p_{3}+A\right)^{2}}=\sum_{p=0}^{\infty} \frac{(p+1)}{(p+A)^{2}},
$$




$$
\begin{aligned}
S_{i}= & \sum_{p=M^{i}}^{M^{i+1}} \frac{(p+1)}{(p+A)^{2}}=\frac{1}{A+M^{i}}+\psi^{(0)}\left(M^{i+1}+A+1\right)-\psi^{(0)}\left(M^{i}+A+1\right) \\
& -(A-1)\left(\psi^{(1)}\left(M^{i}+A\right)-\psi^{(1)}\left(M^{i+1}+A+1\right)\right)
\end{aligned}
$$

where $A$ is a constant (which is set $\frac{m}{a_{2}}$ in the text) and $\psi^{(n)}(z)$ is the so-called polygamma function or $n^{\text {th }}$ derivative of $\ln \Gamma[z]$. There are well-known relations satisfied by the polygamma functions and are given by

$$
\begin{aligned}
& \psi^{(m)}(1+z)=\psi^{(m)}(z)+(-1)^{m} m ! z^{-(m+1)} \\
& \psi^{(0)}(z) \sim \ln z-\frac{1}{2 z}+\ldots, \quad z \rightarrow \infty, \quad|\arg z|<\pi \\
& \psi^{(n)}(z) \sim(-1)^{n-1}\left[\frac{(n-1) !}{z^{n}}+\frac{n !}{2 z^{n+1}}+\ldots\right], \quad z \rightarrow \infty, \quad|\arg z|<\pi, \quad n \geq 1,(B .
\end{aligned}
$$

so that the divergent part of $(\mathrm{B} .17)$ as $M^{i} \gg 1$ can be given as (neglecting all factors of the form constant of convergent factors of the form $1 / M^{i}$ or constants)

$$
S_{i} \sim \kappa[(i+1) \ln M-i \ln M]=\kappa \ln M
$$

hence this does not depend on the scale $i$.

\section{Acknowledgements}

D.O.S. thanks the Perimeter Institute for its hospitality. Discussions with V. Bonzom, R. Gurau and V. Rivasseau are gratefully acknowledged. Research at Perimeter Institute is supported by the Government of Canada through Industry Canada and by the Province of Ontario through the Ministry of Research and Innovation.

\section{References}

[1] B. Zwiebach, "A first course in string theory" (Cambridge University Press, Cambridge UK, 2009).

[2] M. Niedermaier and M. Reuter, "The Asymptotic Safety Scenario in Quantum Gravity," Living Rev. Rel. 9, 5 (2006).

[3] A. Connes, "Noncommutative geometry" (Accademic Press, Boston, 1994).

[4] J. Ambjorn, M. Carfora and A. Marzuoli, "The geometry of dynamical triangulations" (Springer, Heidelberg, 1997).

[5] C. Rovelli, "Quantum gravity" (Cambridge University Press, Cambridge UK, 2004).

[6] A. Connes, M. R. Douglas and A. S. Schwarz, "Noncommutative geometry and matrix theory: Compactification on tori," JHEP 9802, 003 (1998) hep-th/9711162. 
[7] S. Doplicher, K. Fredenhagen and J. E. Roberts, "The Quantum structure of spacetime at the Planck scale and quantum fields," Commun. Math. Phys. 172, 187 (1995) hep-th/0303037.

[8] M. Reuter, "Nonperturbative evolution equation for quantum gravity," Phys. Rev. D 57, 971 (1998) hep-th/9605030.

[9] M. Visser, "Sakharov's induced gravity: A Modern perspective," Mod. Phys. Lett. A 17, 977 (2002) gr-qc/0204062.

[10] R. P. Feynman, F. B. Morinigo, W. G. Wagner and B. Hatfield, "Feynman lectures on gravitation" (Addison-Wesley, Mass., 1995).

[11] M. H. Goroff and A. Sagnotti, "Quantum Gravity At Two Loops," Phys. Lett. B 160, 81 (1985).

M. H. Goroff and A. Sagnotti, "The Ultraviolet Behavior of Einstein Gravity," Nucl. Phys. B 266, 709 (1986).

[12] T. Konopka, F. Markopoulou and L. Smolin, "Quantum Graphity," hep-th/0611197.

[13] P. Di Francesco, P. H. Ginsparg and J. Zinn-Justin, "2-D Gravity and random matrices," Phys. Rept. 254, 1 (1995) arXiv:hep-th/9306153.

[14] J. Ambjorn, B. Durhuus and T. Jonsson, "Three-Dimensional Simplicial Quantum Gravity And Generalized Matrix Models," Mod. Phys. Lett. A 6, 1133 (1991).

[15] M. Gross, "Tensor models and simplicial quantum gravity in > 2-D," Nucl. Phys. Proc. Suppl. 25A, 144 (1992).

[16] N. Sasakura, "Tensor model for gravity and orientability of manifold," Mod. Phys. Lett. A 6, 2613 (1991).

[17] D. V. Boulatov, "A Model of three-dimensional lattice gravity," Mod. Phys. Lett. A 7, 1629 (1992) arXiv:hep-th/9202074;

H. Ooguri, "Topological lattice models in four-dimensions," Mod. Phys. Lett. A 7, 2799 (1992) arXiv:hep-th/9205090.

[18] L. Freidel and K. Krasnov, "Simple spin networks as Feynman graphs," J. Math. Phys. 41, 1681 (2000) arXiv:hep-th/9903192.

[19] M. P. Reisenberger and C. Rovelli, "Space-time as a Feynman diagram: The Connection formulation," Class. Quant. Grav. 18, 121 (2001) gr-qc/0002095.

[20] L. Freidel, "Group field theory: An overview," Int. J. Theor. Phys. 44, 1769 (2005) arXiv:hep-th/0505016.

[21] D. Oriti, "The group field theory approach to quantum gravity," arXiv:gr-qc/0607032. 
[22] L. Freidel, R. Gurau and D. Oriti, "Group field theory renormalization - the 3d case: power counting of divergences," Phys. Rev. D 80, 044007 (2009) arXiv:0905.3772 [hepth]].

[23] J. Magnen, K. Noui, V. Rivasseau and M. Smerlak, "Scaling behavior of threedimensional group field theory," Class. Quant. Grav. 26, 185012 (2009) arXiv:0906.5477 [hep-th]].

[24] J. Ben Geloun, J. Magnen and V. Rivasseau, "Bosonic Colored Group Field Theory," Eur. Phys. J. C 70, 1119 (2010) arXiv:0911.1719 [hep-th]].

[25] J. Ben Geloun, T. Krajewski, J. Magnen and V. Rivasseau, "Linearized Group Field Theory and Power Counting Theorems," Class. Quant. Grav. 27, 155012 (2010) arXiv:1002.3592 [hep-th]].

[26] T. Krajewski, J. Magnen, V. Rivasseau, A. Tanasa and P. Vitale, "Quantum Corrections in the Group Field Theory Formulation of the EPRL/FK Models," Phys. Rev. D 82, 124069 (2010) [arXiv:1007.3150 [gr-qc]].

[27] J. Ben Geloun, R. Gurau and V. Rivasseau, "EPRL/FK Group Field Theory," Europhys. Lett. 92, 60008 (2010) [arXiv:1008.0354 [hep-th]].

[28] V. Bonzom and M. Smerlak, "Bubble divergences from cellular cohomology," Lett. Math. Phys. 93, 295 (2010) arXiv:1004.5196 [gr-qc]].

[29] R. Gurau, "Colored Group Field Theory," Commun. Math. Phys. 304, 69 (2011) arXiv:0907.2582 [hep-th]].

[30] R. Gurau, "Lost in Translation: Topological Singularities in Group Field Theory," Class. Quant. Grav. 27, 235023 (2010) arXiv:1006.0714 [hep-th]].

[31] J. Ben Geloun and V. Bonzom, "Radiative corrections in the Boulatov-Ooguri tensor model: The 2-point function," Int. J. Theor. Phys. 50, 2819 (2011) arXiv:1101.4294 [hep-th]].

[32] D. Oriti and L. Sindoni, "Towards classical geometrodynamics from Group Field Theory hydrodynamics," New J. Phys. 13, 025006 (2011) [arXiv:1010.5149 [gr-qc]].

[33] A. Baratin, F. Girelli and D. Oriti, "Diffeomorphisms in group field theories," Phys. Rev. D 83, 104051 (2011) arXiv:1101.0590 [hep-th]].

[34] J. Ben Geloun, "Ward-Takahashi identities for the colored Boulatov model," J. Phys. A 44, 415402 (2011) arXiv:1106.1847 [hep-th]].

[35] J. Ben Geloun, "Classical Group Field Theory," J. Math. Phys. 53, 022901 (2012) arXiv:1107.3122 [hep-th]].

[36] R. Gurau, "The 1/N expansion of colored tensor models," Annales Henri Poincare 12, 829-847 (2011). [arXiv:1011.2726 [gr-qc]]. 
[37] R. Gurau, V. Rivasseau, "The 1/N expansion of colored tensor models in arbitrary dimension," Europhys. Lett. 95, 50004 (2011). arXiv:1101.4182 [gr-qc]].

[38] R. Gurau, "The complete 1/N expansion of colored tensor models in arbitrary dimension," Annales Henri Poincare 13, 399 (2012) arXiv:1102.5759 [gr-qc]].

[39] V. Rivasseau, "Towards Renormalizing Group Field Theory," PoS C NCFG2010, 004 (2010) arXiv:1103.1900 [gr-qc]].

[40] R. Gurau and J. P. Ryan, "Colored Tensor Models - a review," SIGMA 8, 020 (2012) arXiv:1109.4812 [hep-th]].

[41] V. Bonzom, R. Gurau, A. Riello and V. Rivasseau, "Critical behavior of colored tensor models in the large N limit," Nucl. Phys. B 853, 174 (2011) arXiv:1105.3122 [hep-th]].

[42] R. Gurau, "A generalization of the Virasoro algebra to arbitrary dimensions," Nucl. Phys. B 852, 592 (2011) arXiv:1105.6072 [hep-th]].

[43] V. Bonzom, R. Gurau and V. Rivasseau, "The Ising Model on Random Lattices in Arbitrary Dimensions," Phys. Lett. B 711, 88 (2012) [arXiv:1108.6269 [hep-th]].

[44] R. Gurau, "Universality for Random Tensors," arXiv:1111.0519 [math.PR].

[45] J. Ben Geloun and V. Rivasseau, "A Renormalizable 4-Dimensional Tensor Field Theory," arXiv:1111.4997 [hep-th].

[46] V. Rivasseau, "Quantum Gravity and Renormalization: The Tensor Track," AIP Conf. Proc. 1444, 18 (2011) arXiv:1112.5104 [hep-th]].

[47] V. Rivasseau, "Non-commutative renormalization," arXiv:0705.0705 [hep-th].

[48] H. Grosse and R. Wulkenhaar, "Renormalisation of phi**4 theory on noncommutative $\mathrm{R}^{* *} 4$ in the matrix base," Commun. Math. Phys. 256, 305 (2005) arXiv:hepth/0401128.

[49] V. Rivasseau, F. Vignes-Tourneret and R. Wulkenhaar, "Renormalization of noncommutative phi**4-theory by multi-scale analysis," Commun. Math. Phys. 262, 565 (2006) arXiv:hep-th/0501036.

[50] R. Gurau, J. Magnen, V. Rivasseau and F. Vignes-Tourneret, "Renormalization of noncommutative phi**4(4) field theory in x space," Commun. Math. Phys. 267, 515 (2006) arXiv:hep-th/0512271.

[51] R. Gurau, J. Magnen, V. Rivasseau and A. Tanasa, "A translation-invariant renormalizable non-commutative scalar model," Commun. Math. Phys. 287, 275 (2009) arXiv:0802.0791 [math-ph]].

[52] V. Rivasseau, "From perturbative to constructive renormalization," Princeton series in physics (Princeton Univ. Pr., Princeton, 1991). 
[53] G. Gallavotti and F. Nicolo, "Renormalization theory in four-dimensional scalar fields. I," Commun. Math. Phys. 100, 545 (1985).

[54] R. Gurau, "Topological Graph Polynomials in Colored Group Field Theory," Annales Henri Poincare 11, 565 (2010) arXiv:0911.1945 [hep-th]].

[55] S. Lins, "Gems, Computers and Attractors for 3-Manifolds," Series on Knots and Everything, Vol. 5sss (World Scientific, Singapore, 1995).

[56] M. Ferri and C. Gagliardi, "Cristallisation moves," Pacific Journal of Mathematics 100, 85-103 (1982).

[57] T. Filk, "Divergencies in a field theory on quantum space," Phys. Lett. B 376 (1996) 53-58.

[58] J. Ben Geloun, R. Gurau and V. Rivasseau, "Vanishing beta function for GrosseWulkenhaar model in a magnetic field," Phys. Lett. B 671, 284 (2009) arXiv:0805.4362 [hep-th]].

[59] T. Krajewski, V. Rivasseau and F. Vignes-Tourneret, "Topological graph polynomials and quantum field theory. Part II. Mehler kernel theories," Annales Henri Poincare 12, 483 (2011) arXiv:0912.5438 [math-ph]]. 